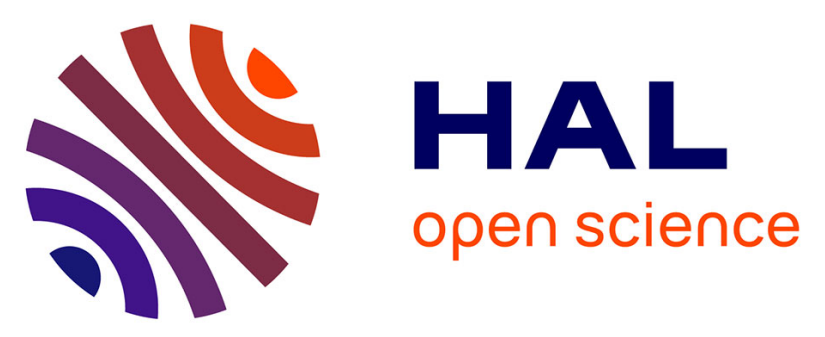

\title{
Two-mica rhyolitic tephra in the East Pisco Basin (Peru): new age and dispersion constraints for the eruptions of the Eastern Cordillera of Central Andes
}

Giulia Bosio, Anna Gioncada, Claudio Di Celma, Igor Maria Villa, Michel

Pichavant, Mario Urbina, Giovanni Bianucci

\section{To cite this version:}

Giulia Bosio, Anna Gioncada, Claudio Di Celma, Igor Maria Villa, Michel Pichavant, et al.. Two-mica rhyolitic tephra in the East Pisco Basin (Peru): new age and dispersion constraints for the eruptions of the Eastern Cordillera of Central Andes. Bulletin of Volcanology, 2020, 82 (6), 10.1007/s00445020-1373-y . insu-02859932

\section{HAL Id: insu-02859932 \\ https://hal-insu.archives-ouvertes.fr/insu-02859932}

Submitted on 15 Feb 2021

HAL is a multi-disciplinary open access archive for the deposit and dissemination of scientific research documents, whether they are published or not. The documents may come from teaching and research institutions in France or abroad, or from public or private research centers.
L'archive ouverte pluridisciplinaire HAL, est destinée au dépôt et à la diffusion de documents scientifiques de niveau recherche, publiés ou non, émanant des établissements d'enseignement et de recherche français ou étrangers, des laboratoires publics ou privés. 


\title{
Two-mica rhyolitic tephra in the East Pisco Basin (Peru): new age and dispersion constraints for the eruptions of the Eastern Cordillera of Central Andes
}

\author{
Giulia Bosio ${ }^{1,2}$ \& Anna Gioncada ${ }^{1}$ \& Claudio Di Celma ${ }^{3}$ \& Igor Maria Villa ${ }^{2,4,5}$ \& Michel Pichavant ${ }^{6}$ \& Mario \\ Urbina $^{7}$ \& Giovanni Bianucci ${ }^{1}$
}

\begin{abstract}
Two-mica-biotite and muscovite-volcanics are particularly rare in the geological record. One of the several dozen volcanic ash layers from Central Andes volcanoes found in the upper Miocene marine succession of the Pisco Formation (Ica Desert, Peru) contains juvenile biotite and muscovite, sillimanite/andalusite, feldspars, and rhyolitic glass. ${ }^{39} \mathrm{Ar}-{ }^{40} \mathrm{Ar}$ dating on biotite and muscovite concordantly constrain an age of $7.96 \mathrm{Ma}$ for this two-mica ash layer. A second tephra in the Pisco Formation has a similar biotite composition and an age between 7.45 and $6.93 \mathrm{Ma}$. The peculiar mineral assemblage and the chemical composition of biotite indicate a strongly peraluminous composition of the erupted magmas and, together with the ${ }^{39} \mathrm{Ar}-{ }^{40} \mathrm{Ar}$ ages, suggest to consider a correlation of these ash layers to the eruptions of the Miocene Macusani (Peru) or Morococala (Bolivia) volcanic complexes in the Eastern Cordillera of Central Andes. The major and trace element composition of glass supports the correlation with Macusani. A prove-nance from Morococala seems less likely given the large distances involved. These results provide new data on the volcanic activity of the Eastern Cordillera revealing ash that dispersed to over $500 \mathrm{~km}$ to the west, in the forearc marine basins. This finding highlights that the exhumed forearc East Pisco Basin is highly promising as an archive of distal ash for the reconstruction of the volcanic activity of Central Andes during the Miocene silicic flare-ups.
\end{abstract}

Keywords Strongly peraluminous tephra ${ }^{\cdot 39} \mathrm{Ar}-{ }^{40} \mathrm{Ar}$ ages ${ }^{\circ}$ Miocene ${ }^{\circ}$ Central Andes ${ }^{\bullet}$ Macusani ${ }^{\bullet}$ Morococala

Editorial responsibility: V.S. Kamenetsky

* Giulia Bosio

giulia.bosio.giulia@gmail.com

Anna Gioncada

anna.gioncada@unipi.it

Claudio Di Celma

claudio.dicelma@unicam.it

Igor Maria Villa

igor.villa@unimib.it; igor.villa@geo.unibe.ch

Michel Pichavant

pichavan@cnrs-orleans.fr

Mario Urbina

mariourbina01@hotmail.com

Giovanni Bianucci

giovanni.bianucci@unipi.it
1 Dipartimento di Scienze della Terra, Università di Pisa, 56126 Pisa, Italy

2 Dipartimento di Scienze dell'Ambiente e della Terra, Università degli Studi di Milano-Bicocca, 20126 Milan, Italy

3

Scuola di Scienze e Tecnologie, Università di Camerino, 62032 Camerino, Italy

4 Institut für Geologie, Universität Bern, 3012 Bern, Switzerland

5 Centro Universitario Datazioni e Archeometria, Università degli Studi di Milano-Bicocca, 20126 Milan, Italy

6 Université d'Orléans/CNRS/ISTO/BRGM, UMR 7327, 1A rue de la Férollerie, 45100 Orléans, France

7 Departamento de Paleontología de Vertebrados, Museo de Historia Natural, Universidad Nacional Mayor de San Marcos, Lima 1, Peru 
Strongly peraluminous (SP) volcanics (in the sense of Miller 1985: $\mathrm{Al}_{2} \mathrm{SiO}_{5-}$, two mica-, garnet- or cordierite-bearing "granitic" rocks) are particularly rare in the geological record. One of the largest peraluminous volcanic provinces is in the Eastern Cordillera of the Central Andes. Here, tectonomagmatic conditions responsible for generating crustal melts of strongly peraluminous composition prevailed for several million years in late Miocene (Sandeman et al. 1997; Caffe et al. 2012). Although the Cenozoic marine sediments of the offshore and emerged forearc basins of southern Peru host many tuff layers representing distal volcanic ash of Central Andes eruptions, dispersed westwards by easterly winds (Pouclet et al. 1990; Hart and Miller 2006; Bosio et al. 2019), no correlations with the Eastern Cordillera activity have been proposed up to now.

In the East Pisco Basin (southern Peru), the Miocene Pisco Formation hosts many undisturbed volcanic ash layers in diatomaceous sedimentary successions (Di Celma et al. 2017; Bosio et al. 2020b). Most of them, based on glass and biotite composition and mineral assemblage, are calc-alkaline dacites and rhyolites and subordinate andesites (Bosio et al. 2019) representing the subduction-related activity of the Western Cordillera (Mamani et al. 2010). A few, instead, show biotite composition pointing to the siderophyllite endmember, sug-gesting a peraluminous nature of the erupted magma (Bosio et al. 2019). One of these latter ash layers is a two-mica- biotite and muscovite-rhyolitic tephra.

In this paper, we provide field and petrochemical descriptions of volcanic ash and age determinations, and discuss the possible finding of Eastern Cordillera ash in the forearc East Pisco Basin. These results add new information about the volcanic activity of the Eastern Cordillera of Central Andes and reveal two major eruptions of strongly peraluminous magmas at 7-8 Ma that dispersed volcanic products up to hundreds of kilometers to the west, reaching the coastal forearc basins.

\section{Geological and volcanological framework}

Since the Mesozoic, the western margin of South America has been influenced by the subduction of the oceanic Nazca/ Farallon Plate underneath the continental South American
Fig. 1 Geographical setting and Eastern Cordillera volcanism of the Central Andes. The orange star shows the East Pisco Basin (EPB) location. Blue stars show the main Eastern Cordillera volcanic fields $(\mathrm{MAC}=$ Macusani; MOR = Morococala) . CC stays for Coastal Cordillera, PC for Pre-Cordillera, WC for Western Cordillera, A for Altipiano, EC for Eastern Cordillera, and SA for Subandean zone

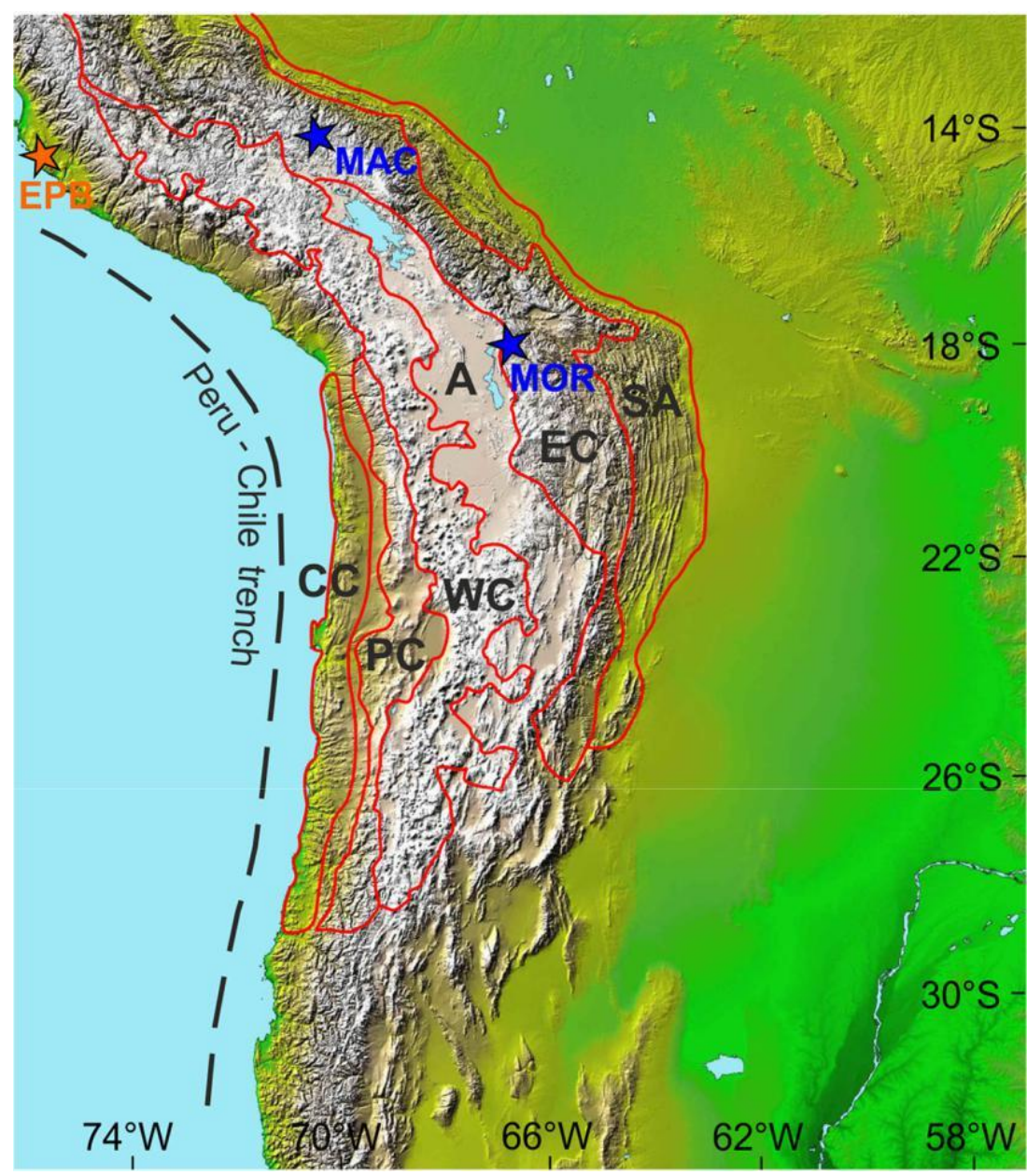




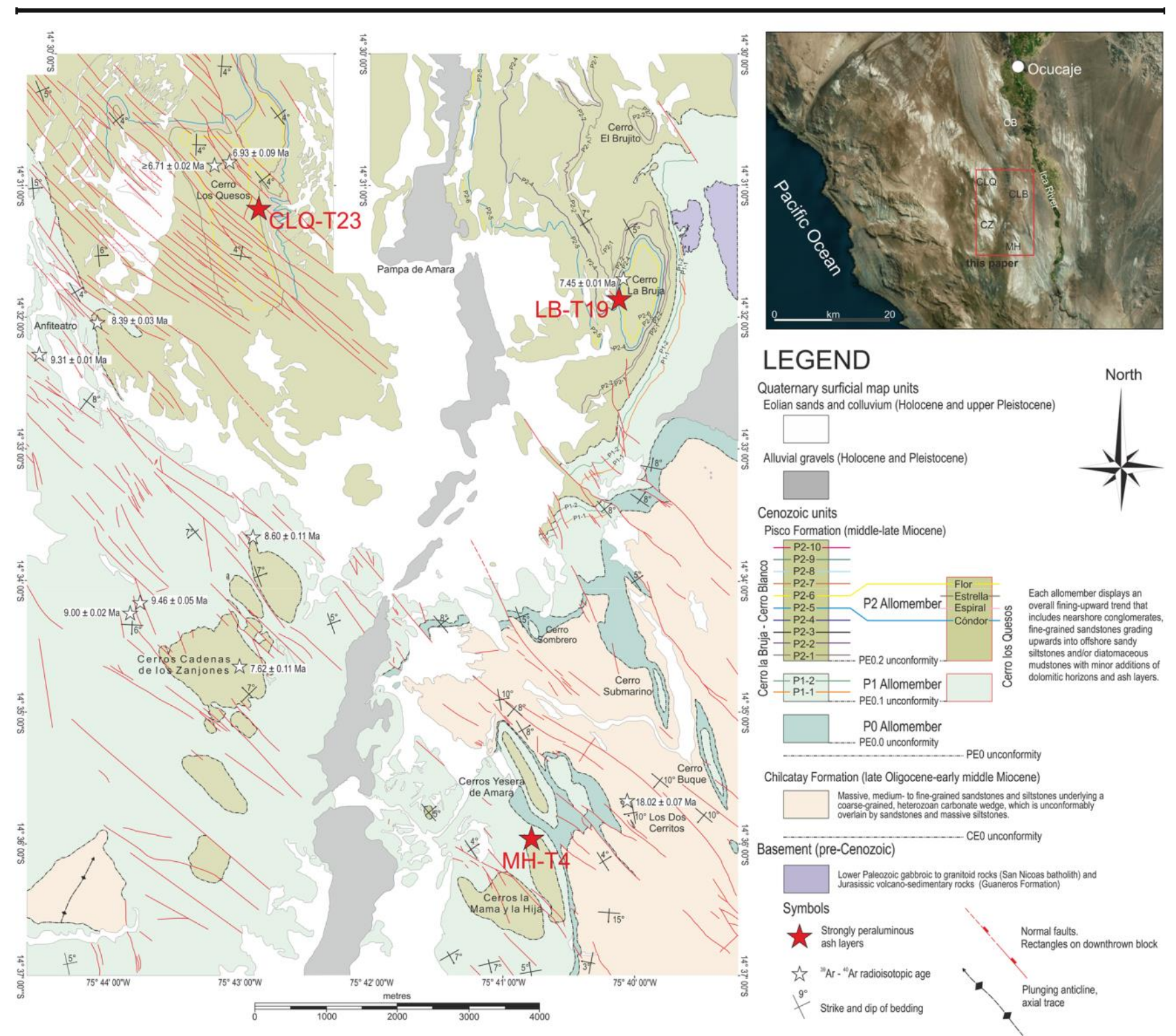

Fig. 2 Geological map of the study area. Geological map of the studied localities in the western side of the Ica River and geographic position of SP tephra mentioned in this paper (red stars) modified from Di Celma et al. (2018) and Bosio et al. (2020b)

Fig. 3 Field photo of Cerros la Mama y la Hija. PE0.2 unconformity (i.e., the unconformity found at the base of the P2 allomember) (red continuous line) and MH-T4 tephra layer (yellow dashed line) at the locality of Cerros la Mama y la Hija

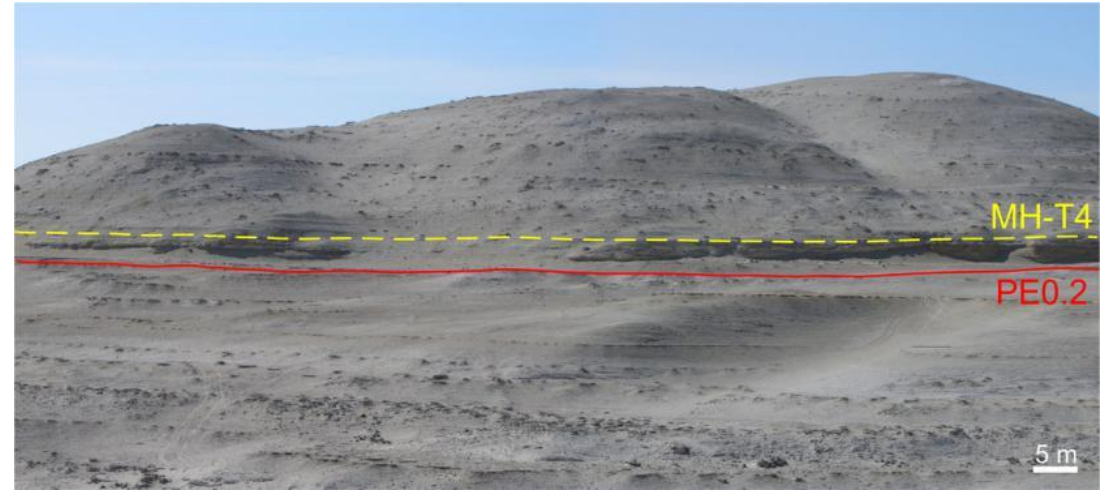



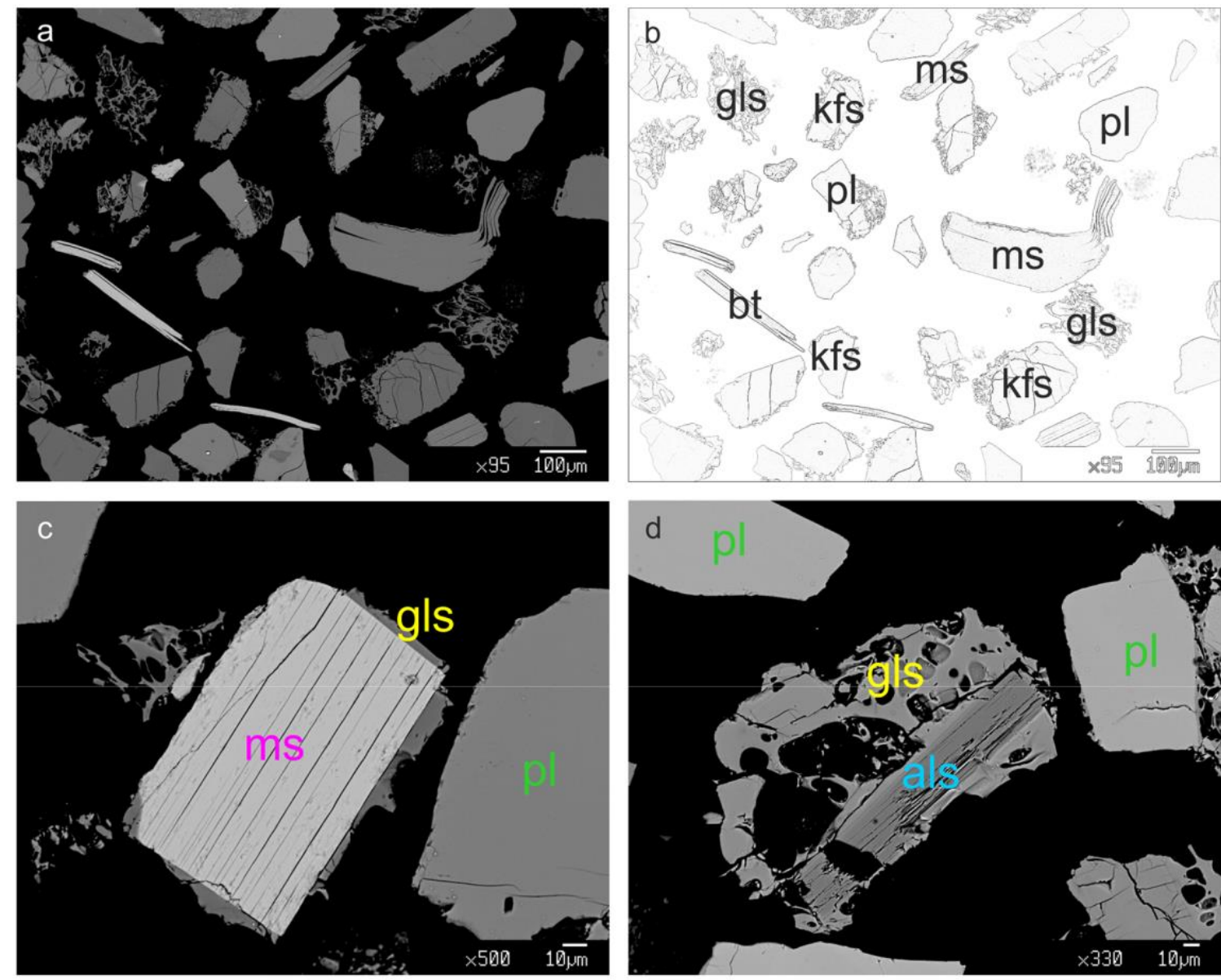

e

Particle diameter $(\phi)$

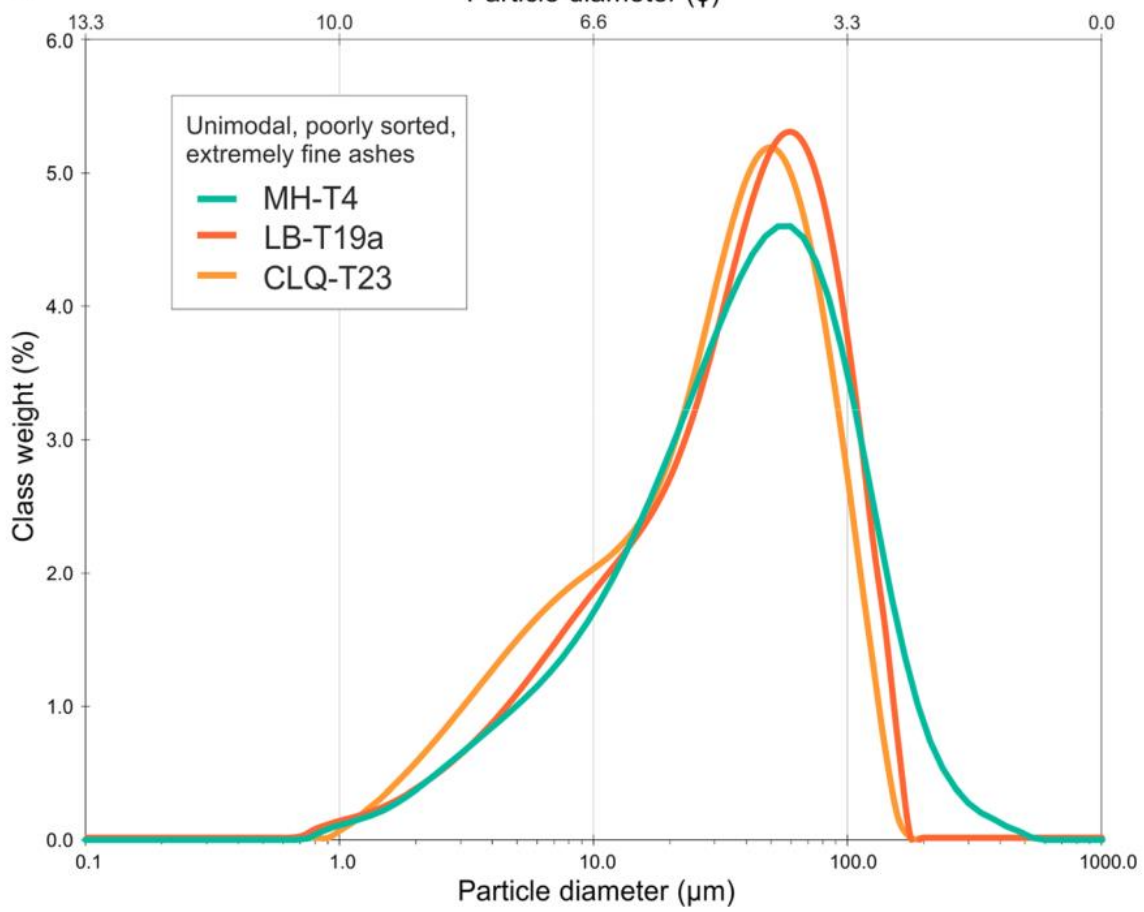


R Fig. 4 BSE images and grain-size distribution of peraluminous tephra. a BSE image of 250-500 $\mu \mathrm{m}$ fraction of MH-T4 tephra. b Contours of the crystals shown in Fig. 4a showing biotite (bt), plagioclase (pl), K-feldspar (Kfs), and muscovite (ms) phenocrysts, and glass shards (gls). c BSE image of a well-preserved muscovite phenocryst surrounded by volcanic glass in the MH-T4 sample. d BSE image of an $\mathrm{Al}_{2} \mathrm{SiO}_{5}$ mineral inclusion in a high-vesiculated glass shard in the MH-T4 sample. e Grainsize distribution curves of MH-T4 ash layer and the tephra pairs CLQT23 and LB-T19. Distribution is unimodal; particle diameter is extremely fine ash. Particle size is shown as both micrometers and $\phi$

Plate. In the Central Andes, this subduction was responsible for the production of calc-alkaline metaluminous magmas along the western magmatic arcs forming the Western Cordillera (Baker and Francis 1978; de Silva and Francis 1991; Trumbull et al. 2006; Mamani et al. 2010). On the eastern side of the Central Andes orogen, instead, the peraluminous and S-type volcanic and intrusive magmatic units emplaced since $25 \mathrm{Ma}$ indicate that the Eastern Cordillera magmatism is not related to subduction and was the result of extensive crustal melting (Trumbull et al. 2006). Confirming this petrogenetic distinction, the extensive fractional crystallization of the Eastern Cordillera crustal-derived magmas led to $\mathrm{Sn}$ and $\mathrm{W}$ enrichment (Mlynarczyk and Williams-Jones 2005), while in the western Cordillera, predominantly mantle-derived, metaluminous magmas led to porphyry $\mathrm{Cu}-\mathrm{Au}-\mathrm{Mo}$ and epithermal ore deposits (Rosenbaum et al. 2005). Examples of the crustal-derived magmatic units of the Eastern Cordillera emplaced from $14^{\circ} \mathrm{S}$ to $26^{\circ} \mathrm{S}$ (see Fig. 1) are the Kari Kari ignimbrite (Schneider 1987), the Macusani volcanics (Noble et al. 1984; Pichavant et al. 1988a, b), the Morococala Meseta ig-nimbrites (Morgan et al. 1998), Revancha dyke (Sandeman and Clark 2003), Cayconi volcanic field (Sandeman and Clark 2004), and Los Frailes ignimbrites (Kay et al. 2010).

Volcanic ash layers linked to the activity of the Central Andes (Central Volcanic Zone) have been found along the Peruvian and Chilean coast (Pouclet et al. 1990; Hart and Miller 2006; Breitkreuz et al. 2014; Bosio et al. 2019), in the Coastal Cordillera and in the forearc basins formed during the Cenozoic (Travis et al. 1976; Thornburg and Kulm 1981). These products represent the distal ash of large explosive erup-tions, which may have been dispersed westwards by south-southeasterly winds, similar to the products of the Huaynaputina eruption, the largest explosive event in historical times (1600 AD) in Peru (Thouret et al. 1999; de Silva and Zielinski 1998).

One of the forearc basins, the East Pisco Basin now exposed in the Ica Desert of Peru, hosts one of the most world-renowned marine vertebrate Fossil-Lagerstätte (Esperante et al. 2015; Bianucci et al. 2016a, b, 2018).

The Miocene Pisco Formation is the youngest unit of the East Pisco Basin marine fill and is mainly composed of diatomaceous siltstones and sandstones, interposed by frequent tephra layers (Dunbar et al. 1990). Di Celma et al. (2017) divided this formation in three main allomembers, P0, $\mathrm{P} 1$, and $\mathrm{P} 2$, from the oldest to the youngest, bounded by regionally extensive intraformational unconformities. $\mathrm{P} 1$ and P2 allomembers are composed of yellow sandstones at the base and white diatomaceous siltstone at the top. Along the western side of the Ica River, the P0 allomember was deposited in between 14.8 and 12.4 Ma (Bosio et al. 2020a), the P1 allomember between 9.5 and $8.6 \mathrm{Ma}$, whereas the P2 allomember was deposited from 8.4 to at least 6.7 Ma (Bosio et al. 2020b). Volcanic ash layers are mainly preserved in the diatomaceous mudstone portions of the marine succes-sions of the P1 and P2 allomembers.

\section{Methods}

Among more than 200 samples of volcanic ash layers collect-ed in the East Pisco Basin succession during the fieldwork from 2014 to 2016, this work focuses on three ash samples from the localities of Cerros la Mama y la Hija, Cerro los Quesos, and Cerro la Bruja (Fig. 2). Samples were collected at the base of the ash layer in order to avoid any possible reworking and bioturbation affecting the top of the layer. The surface of the outcrop was discarded to avoid weathered or extraneous grains. Before compositional and ${ }^{39} \mathrm{Ar}-{ }^{40} \mathrm{Ar}$ dating analyses, the samples were inspected under a stereomicroscope, and smear slides were studied under an optical microscope for petrographic features. The ash layers were considered as the primary deposition of tephra on the basis of a percentage of volcanic components (glass shards and juvenile loose crystals) greater than 95 vol\% (Griggs et al. 2014; Tada et al. 2015).

Grain-size analyses were performed with the Malvern Mastersizer 2000E ${ }^{\mathrm{TM}}$ Laser Granulometer and processed with the grain-size analysis program GRADISTAT (Blott and Pye 2001) at the Università di Milano-Bicocca (Dipartimento di Scienze dell'Ambiente e della Terra, Milano, Italy). The ash samples were wet sieved with meshes of 500, 250, 125, and $63 \mu \mathrm{m}$, and the $125-250 \mu \mathrm{m}$ and $250-500 \mu \mathrm{m}$ fractions were mounted in resin and polished for compositional analyses. SEM-EDS (Scanning Electron Microscope equipped with Electron Dispersion Spectroscopy) microanalysis was carried out at the Università di Pisa (Dipartimento di Scienze della Terra, Pisa, Italy) with a Philips ${ }^{\text {TM }}$ XL30 scanning electron microscope equipped with an Edax Genesis microanalytical device, with 20-kV filament voltage, 5-nA beam current, and ZAF correction. EPMA (Electron Probe Micro-Analysis) analyses for major elements were performed on glass shards, Al-Si minerals, and biotite and muscovite phenocrysts with a JEOL 8200 Superprobe $^{\mathrm{TM}}$ at the Università di Milano (Dipartimento di Scienze della Terra "Ardito Desio," Milano, Italy) (15-kV accelerating voltage, 5-nA beam 
Table 1 EPMA analyses on biotite phenocrysts of MH-T4 tephra

Provenance of samples:

MH-T4 - Pisco Basin

Source:

This work

\begin{tabular}{|c|c|c|c|c|c|c|c|c|c|c|c|c|c|c|c|}
\hline \multirow[t]{2}{*}{ Source: } & \multicolumn{15}{|c|}{ This work } \\
\hline & $1-1$ & $1-2$ & $2-1$ & $2-2$ & $3-1$ & $3-2$ & $4-1$ & $4-2$ & $5-1$ & $5-2$ & $6-1$ & $6-2$ & $7-1$ & $7-2$ & $8-1$ \\
\hline $\mathrm{SiO}_{2} \mathrm{wt} \%$ & 34.83 & 35.32 & 35.76 & 34.94 & 35.79 & 35.06 & 34.73 & 35.61 & 35.42 & 35.15 & 35.98 & 34.92 & 34.96 & 35.47 & 35.72 \\
\hline $\mathrm{TiO}_{2}$ & 3.69 & 3.51 & 2.98 & 3.17 & 3.06 & 3.01 & 3.85 & 3.75 & 3.66 & 3.76 & 3.25 & 3.24 & 3.31 & 3.32 & 3.81 \\
\hline $\mathrm{Al}_{2} \mathrm{O}_{3}$ & 19.25 & 19.33 & 18.85 & 18.36 & 19.67 & 19.54 & 18.86 & 18.90 & 18.75 & 18.81 & 19.23 & 19.18 & 19.39 & 19.60 & 18.92 \\
\hline $\mathrm{FeO}^{\mathrm{T}}$ & 20.71 & 21.23 & 21.93 & 22.24 & 21.16 & 21.30 & 20.73 & 20.85 & 20.64 & 20.84 & 22.17 & 22.33 & 20.84 & 20.76 & 21.02 \\
\hline $\mathrm{MnO}$ & 0.17 & 0.13 & 0.31 & 0.28 & 0.25 & 0.23 & 0.10 & 0.17 & 0.14 & 0.13 & 0.27 & 0.18 & 0.17 & 0.21 & 0.11 \\
\hline $\mathrm{MgO}$ & 6.87 & 6.94 & 5.56 & 5.35 & 6.13 & 6.34 & 7.21 & 7.33 & 7.18 & 7.01 & 5.49 & 5.65 & 6.60 & 6.67 & 7.16 \\
\hline $\mathrm{CaO}$ & bd & bd & 0.02 & $\mathrm{bd}$ & bd & bd & bd & 0.04 & bd & bd & 0.01 & bd & 0.01 & 0.01 & 0.02 \\
\hline $\mathrm{Na}_{2} \mathrm{O}$ & 0.38 & 0.35 & 0.34 & 0.37 & 0.27 & 0.39 & 0.35 & 0.30 & 0.33 & 0.26 & 0.34 & 0.43 & 0.37 & 0.35 & 0.33 \\
\hline $\mathrm{K}_{2} \mathrm{O}$ & 9.05 & 8.88 & 8.94 & 8.93 & 9.14 & 9.13 & 9.04 & 8.98 & 9.21 & 9.14 & 8.94 & 8.76 & 9.20 & 9.08 & 9.12 \\
\hline $\mathrm{F}$ & na & na & na & na & na & na & na & na & na & na & na & na & na & na & na \\
\hline $\mathrm{Cl}$ & 0.10 & 0.09 & 0.16 & 0.17 & 0.05 & 0.05 & 0.15 & 0.14 & 0.17 & 0.18 & 0.18 & 0.17 & 0.07 & 0.06 & 0.11 \\
\hline $\mathrm{ZnO}$ & na & na & na & na & na & na & na & na & na & na & na & na & na & na & na \\
\hline $\mathrm{BaO}$ & 0.11 & 0.20 & 0.13 & 0.09 & 0.09 & 0.06 & 0.20 & 0.09 & 0.02 & 0.00 & 0.06 & 0.00 & 0.16 & 0.14 & 0.10 \\
\hline Total & 95.15 & 95.98 & 94.98 & 93.90 & 95.61 & 95.12 & 95.28 & 96.25 & 95.58 & 95.33 & 95.93 & 94.95 & 95.10 & 95.67 & 96.43 \\
\hline \multicolumn{16}{|c|}{ Ions on the basis of 22 oxygens } \\
\hline Si apfu & 5.35 & 5.38 & 5.52 & 5.48 & 5.46 & 5.40 & 5.34 & 5.40 & 5.41 & 5.38 & 5.49 & 5.40 & 5.38 & 5.41 & 5.41 \\
\hline $\mathrm{Ti}$ & 0.43 & 0.40 & 0.35 & 0.37 & 0.35 & 0.35 & 0.44 & 0.43 & 0.42 & 0.43 & 0.37 & 0.38 & 0.38 & 0.38 & 0.43 \\
\hline $\mathrm{Al}$ & 3.49 & 3.47 & 3.43 & 3.39 & 3.54 & 3.55 & 3.42 & 3.38 & 3.37 & 3.40 & 3.46 & 3.50 & 3.52 & 3.52 & 3.38 \\
\hline AlIV & 2.65 & 2.62 & 2.48 & 2.52 & 2.54 & 2.60 & 2.66 & 2.60 & 2.59 & 2.62 & 2.51 & 2.60 & 2.62 & 2.59 & 2.59 \\
\hline AlvI & 0.83 & 0.85 & 0.95 & 0.87 & 1.00 & 0.94 & 0.75 & 0.78 & 0.78 & 0.78 & 0.95 & 0.90 & 0.90 & 0.93 & 0.78 \\
\hline $\mathrm{Fe}$ & 2.66 & 2.70 & 2.83 & 2.92 & 2.70 & 2.74 & 2.66 & 2.64 & 2.64 & 2.67 & 2.83 & 2.89 & 2.68 & 2.65 & 2.66 \\
\hline Mn & 0.02 & 0.02 & 0.04 & 0.04 & 0.03 & 0.03 & 0.01 & 0.02 & 0.02 & 0.02 & 0.04 & 0.02 & 0.02 & 0.03 & 0.01 \\
\hline $\mathrm{Mg}$ & 1.57 & 1.58 & 1.28 & 1.25 & 1.39 & 1.45 & 1.65 & 1.66 & 1.63 & 1.60 & 1.25 & 1.30 & 1.51 & 1.52 & 1.62 \\
\hline $\mathrm{Ca}$ & bd & bd & bd & bd & bd & bd & bd & 0.01 & bd & $\mathrm{bd}$ & bd & bd & bd & bd & bd \\
\hline $\mathrm{Na}$ & 0.11 & 0.10 & 0.10 & 0.11 & 0.08 & 0.12 & 0.11 & 0.09 & 0.10 & 0.08 & 0.10 & 0.13 & 0.11 & 0.10 & 0.10 \\
\hline $\mathrm{K}$ & 1.77 & 1.73 & 1.76 & 1.79 & 1.78 & 1.79 & 1.77 & 1.74 & 1.79 & 1.79 & 1.74 & 1.73 & 1.81 & 1.77 & 1.76 \\
\hline $\mathrm{Cl}$ & 0.02 & 0.02 & 0.04 & 0.05 & 0.01 & 0.01 & 0.04 & 0.04 & 0.04 & 0.05 & 0.05 & 0.05 & 0.02 & 0.02 & 0.03 \\
\hline $\mathrm{Zn}$ & na & na & na & na & na & na & na & na & na & na & na & na & na & na & na \\
\hline $\mathrm{Ba}$ & 0.01 & 0.01 & 0.01 & 0.01 & 0.01 & $\mathrm{bd}$ & 0.01 & 0.01 & $\mathrm{bd}$ & $\mathrm{bd}$ & $\mathrm{bd}$ & $\mathrm{bd}$ & 0.01 & 0.01 & 0.01 \\
\hline sum & 15.43 & 15.41 & 15.36 & 15.40 & 15.35 & 15.44 & 15.46 & 15.40 & 15.43 & 15.41 & 15.33 & 15.40 & 15.45 & 15.39 & 15.41 \\
\hline $\mathrm{X}$ & 1.89 & 1.84 & 1.87 & 1.91 & 1.86 & 1.91 & 1.89 & 1.84 & 1.89 & 1.86 & 1.84 & 1.86 & 1.93 & 1.88 & 1.86 \\
\hline $\mathrm{Y}$ & 5.51 & 5.55 & 5.44 & 5.45 & 5.48 & 5.52 & 5.53 & 5.52 & 5.49 & 5.50 & 5.44 & 5.49 & 5.50 & 5.50 & 5.51 \\
\hline $\mathrm{MgV}$ & 0.37 & 0.37 & 0.31 & 0.30 & 0.34 & 0.35 & 0.38 & 0.39 & 0.38 & 0.37 & 0.31 & 0.31 & 0.36 & 0.36 & 0.38 \\
\hline
\end{tabular}

Provenance of samples: $\quad$ MH-T4 - Pisco Basin

Source:

This work

\begin{tabular}{llllllllllll}
\hline $8-2$ & $9-1$ & $9-2$ & $10-$ & $10-$ & $11-$ & $11-$ & $12-$ & $12-$ & $13-$ & $13-$ & $14-$ \\
2 & $14-$ & $15-$ & $15-$
\end{tabular}

$\mathrm{SiO}_{2} \mathrm{wt} \%$

$\mathrm{TiO}_{2}$

$\mathrm{FeO}^{\mathrm{T}} \mathrm{O}_{3}$

$\mathrm{MnO}$

$35.53 \quad 34.12$

35.73

35.53
3.72
18.92

$\begin{array}{ll}3.72 & 3.45 \\ 18.92 & 18.54\end{array}$

$20.75 \quad 21.80$

34.96

34.91

34.20

34.62

34.43

$\begin{array}{ll}3.45 & 3.39 \\ 8.54 & 19.0 \\ 21.80 & 21.6 \\ 0.18 & 0.15\end{array}$

$\begin{array}{lllll}34.39 & 34.91 & 34.64 & 34.20 & 34.62 \\ 19.09 & 18.96 & 3.72 & 3.23 & 3.28\end{array}$

$\begin{array}{llll}19.09 & 18.96 & 18.79 & 3.23 \\ 21.66 & 21.01 & 20.68 & 21.73 \\ 0.15 & 0.07 & 0.10 & 0.15\end{array}$

$\begin{array}{lll}3.28 & 34.43 & 34.95 \\ 19.08 & 19.96 & 2.96 \\ 21.52 & 21.15 & 20.88\end{array}$

$\begin{array}{ll}21.52 & 21.15 \\ 0.17 & 0.22\end{array}$


Table 1 (continued)

\begin{tabular}{|c|c|c|c|c|c|c|c|c|c|c|c|c|c|c|c|}
\hline $\mathrm{MgO}$ & 7.18 & 6.44 & 6.21 & 7.30 & 7.33 & 6.20 & 6.06 & 6.21 & 6.10 & 7.22 & 7.20 & 7.23 & 7.18 & 6.66 & 6.66 \\
\hline $\mathrm{CaO}$ & 0.02 & 0.01 & 0.03 & 0.01 & bd & 0.03 & bd & 0.02 & 0.01 & 0.04 & 0.03 & 0.01 & 0.03 & 0.01 & 0.04 \\
\hline $\mathrm{Na}_{2} \mathrm{O}$ & 0.32 & 0.28 & 0.34 & 0.33 & 0.29 & 0.33 & 0.29 & 0.39 & 0.31 & 0.36 & 0.32 & 0.34 & 0.33 & 0.36 & 0.33 \\
\hline $\mathrm{K}_{2} \mathrm{O}$ & 9.01 & 9.11 & 9.12 & 9.15 & 9.12 & 8.85 & 8.89 & 9.06 & 9.06 & 9.10 & 9.15 & 9.06 & 9.07 & 9.04 & 9.09 \\
\hline $\mathrm{F}$ & na & na & na & na & na & na & na & na & na & na & na & na & na & na & na \\
\hline $\mathrm{Cl}$ & 0.14 & 0.10 & 0.13 & 0.15 & 0.14 & 0.17 & 0.18 & 0.05 & 0.05 & 0.17 & 0.17 & 0.16 & 0.18 & 0.09 & 0.08 \\
\hline $\mathrm{ZnO}$ & na & na & na & na & na & na & na & na & na & na & na & na & na & na & na \\
\hline $\mathrm{BaO}$ & 0.35 & 0.09 & 0.07 & 0.09 & 0.13 & 0.00 & 0.10 & 0.02 & 0.00 & 0.04 & 0.13 & 0.35 & 0.04 & 0.17 & 0.06 \\
\hline Total & 96.05 & 94.12 & 95.15 & 95.77 & 94.98 & 94.18 & 94.19 & 94.64 & 94.31 & 94.51 & 94.65 & 93.86 & 93.23 & 94.55 & 94.29 \\
\hline \multicolumn{16}{|c|}{ Ions on the basis of 22 oxygens } \\
\hline Si apfu & 5.41 & 5.34 & 5.39 & 5.34 & 5.34 & 5.33 & 5.39 & 5.33 & 5.41 & 5.39 & 5.37 & 5.34 & 5.32 & 5.38 & 5.41 \\
\hline $\mathrm{Ti}$ & 0.43 & 0.41 & 0.39 & 0.43 & 0.43 & 0.38 & 0.38 & 0.36 & 0.34 & 0.42 & 0.43 & 0.45 & 0.43 & 0.39 & 0.36 \\
\hline $\mathrm{Al}$ & 3.39 & 3.42 & 3.47 & 3.42 & 3.41 & 3.54 & 3.50 & 3.64 & 3.59 & 3.38 & 3.41 & 3.36 & 3.41 & 3.50 & 3.50 \\
\hline AlIV & 2.59 & 2.66 & 2.61 & 2.66 & 2.66 & 2.67 & 2.61 & 2.67 & 2.59 & 2.61 & 2.63 & 2.66 & 2.68 & 2.62 & 2.59 \\
\hline AlVI & 0.80 & 0.77 & 0.86 & 0.75 & 0.75 & 0.87 & 0.89 & 0.97 & 1.00 & 0.77 & 0.79 & 0.70 & 0.73 & 0.88 & 0.91 \\
\hline $\mathrm{Fe}$ & 2.64 & 2.86 & 2.79 & 2.69 & 2.67 & 2.83 & 2.80 & 2.74 & 2.70 & 2.62 & 2.60 & 2.68 & 2.68 & 2.68 & 2.67 \\
\hline $\mathrm{Mn}$ & 0.01 & 0.02 & 0.02 & 0.01 & 0.01 & 0.02 & 0.02 & 0.03 & 0.03 & 0.02 & 0.01 & 0.02 & 0.01 & 0.03 & 0.03 \\
\hline $\mathrm{Mg}$ & 1.63 & 1.50 & 1.43 & 1.66 & 1.68 & 1.44 & 1.41 & 1.43 & 1.41 & 1.66 & 1.65 & 1.69 & 1.68 & 1.54 & 1.54 \\
\hline $\mathrm{Ca}$ & bd & bd & 0.01 & bd & bd & 0.01 & bd & bd & bd & 0.01 & 0.01 & bd & 0.01 & bd & 0.01 \\
\hline $\mathrm{Na}$ & 0.10 & 0.09 & 0.10 & 0.10 & 0.09 & 0.10 & 0.09 & 0.12 & 0.09 & 0.11 & 0.10 & 0.10 & 0.10 & 0.11 & 0.10 \\
\hline K & 1.75 & 1.82 & 1.79 & 1.78 & 1.79 & 1.76 & 1.77 & 1.79 & 1.79 & 1.79 & 1.80 & 1.81 & 1.82 & 1.78 & 1.80 \\
\hline $\mathrm{Cl}$ & 0.04 & 0.03 & 0.03 & 0.04 & 0.04 & 0.04 & 0.05 & 0.01 & 0.01 & 0.04 & 0.04 & 0.04 & 0.05 & 0.02 & 0.02 \\
\hline $\mathrm{Zn}$ & na & na & na & na & na & na & na & na & na & na & na & na & na & na & na \\
\hline $\mathrm{Ba}$ & 0.02 & 0.01 & bd & 0.01 & 0.01 & bd & 0.01 & bd & bd & bd & 0.01 & 0.02 & bd & 0.01 & bd \\
\hline sum & 15.41 & 15.50 & 15.43 & 15.47 & 15.47 & 15.45 & 15.41 & 15.45 & 15.39 & 15.45 & 15.44 & 15.51 & 15.50 & 15.44 & 15.43 \\
\hline X & 1.87 & 1.91 & 1.90 & 1.89 & 1.89 & 1.86 & 1.86 & 1.91 & 1.88 & 1.91 & 1.91 & 1.93 & 1.92 & 1.90 & 1.90 \\
\hline Y & 5.51 & 5.56 & 5.49 & 5.54 & 5.55 & 5.54 & 5.50 & 5.52 & 5.49 & 5.50 & 5.49 & 5.54 & 5.53 & 5.51 & 5.51 \\
\hline $\mathrm{MgV}$ & 0.38 & 0.34 & 0.34 & 0.38 & 0.39 & 0.34 & 0.33 & 0.34 & 0.34 & 0.39 & 0.39 & 0.39 & 0.39 & 0.36 & 0.37 \\
\hline
\end{tabular}

The first number is the crystal and the second one represents the core (1) or the rim (2). X is K + Na + Ba and Y is AlVI+Mg + Fe + Mn + Ti. Abbreviations: "na" stands for "not analyzed" and "bd" stands for "below detection" 
Table 2 EPMA analyses on biotite phenocrysts of MH-T4 tephra (continuation)

Provenance of samples:

Source:

MH-T4 - Pisco Basin

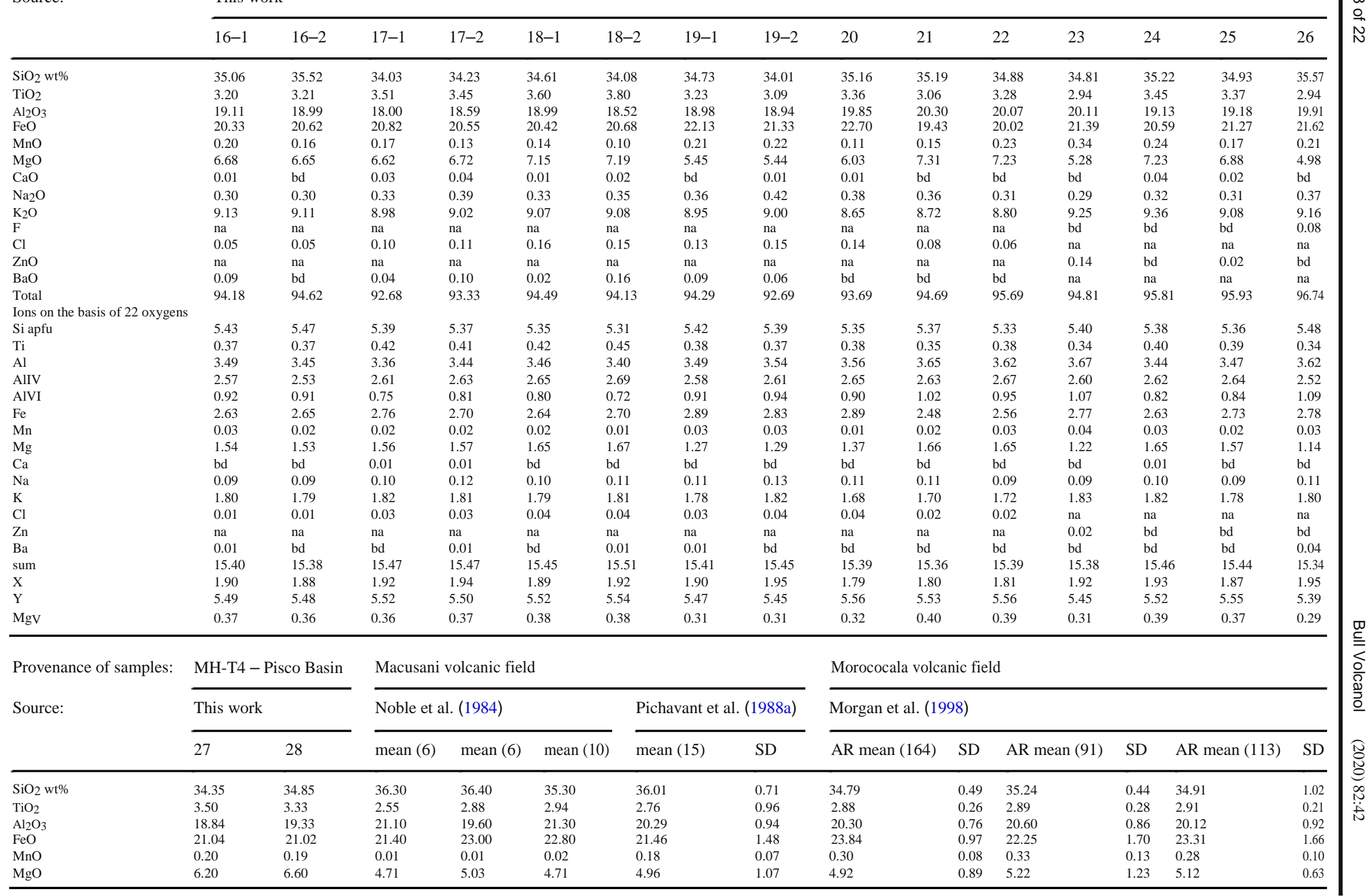


Table 2 (continued)

\begin{tabular}{|c|c|c|c|c|c|c|c|c|c|c|c|c|c|}
\hline $\mathrm{CaO}$ & bd & $\mathrm{bd}$ & $\mathrm{bd}$ & bd & bd & 0.06 & 0.11 & bd & bd & bd & bd & bd & $\mathrm{bd}$ \\
\hline $\mathrm{Na}_{2} \mathrm{O}$ & 0.31 & 0.30 & 0.37 & 0.35 & 0.36 & 0.49 & 0.12 & 0.40 & 0.02 & 0.40 & 0.03 & 0.40 & 0.03 \\
\hline $\mathrm{K}_{2} \mathrm{O}$ & 9.40 & 9.43 & 9.12 & 8.63 & 8.84 & 9.00 & 0.36 & 8.73 & 0.19 & 8.83 & 0.20 & 8.67 & 0.26 \\
\hline $\mathrm{F}$ & bd & bd & 0.01 & 0.01 & 0.02 & 2.45 & 0.93 & 2.54 & 0.71 & 3.47 & 0.71 & 3.39 & 0.96 \\
\hline $\mathrm{Cl}$ & na & na & 0.10 & 0.17 & 0.10 & na & na & 0.11 & 0.03 & 0.12 & 0.03 & 0.12 & 0.03 \\
\hline $\mathrm{ZnO}$ & 0.18 & bd & na & na & na & 0.13 & 0.18 & 0.15 & 0.04 & 0.15 & 0.04 & 0.12 & 0.03 \\
\hline $\mathrm{BaO}$ & na & na & na & na & na & 0.35 & 0.11 & 0.05 & 0.02 & 0.05 & 0.02 & 0.07 & 0.06 \\
\hline Total & 94.07 & 95.11 & 93.69 & 94.69 & 95.69 & 96.14 & 2.02 & 97.83 & - & 98.02 & - & 97.98 & - \\
\hline \multicolumn{14}{|c|}{ Ions on the basis of 22 oxygens } \\
\hline Si apfu & 5.37 & 5.37 & 5.51 & 5.54 & 5.36 & 5.42 & 0.24 & 5.58 & - & 5.87 & - & 6.09 & - \\
\hline $\mathrm{Ti}$ & 0.41 & 0.39 & 0.29 & 0.33 & 0.34 & 0.31 & 0.11 & 0.35 & - & 0.36 & - & 0.38 & - \\
\hline $\mathrm{Al}$ & 3.47 & 3.51 & 3.77 & 3.51 & 3.81 & 3.60 & 0.27 & 3.84 & - & 4.04 & - & 4.14 & - \\
\hline AlIV & 2.63 & 2.63 & 2.49 & 2.46 & 2.64 & 2.58 & - & 2.42 & - & 2.13 & - & 1.91 & - \\
\hline AIVI & 0.84 & 0.88 & 1.28 & 1.05 & 1.17 & 1.02 & - & 1.41 & - & 1.91 & - & 2.23 & - \\
\hline $\mathrm{Fe}$ & 2.75 & 2.71 & 2.72 & 2.93 & 2.89 & 2.70 & 0.15 & 3.20 & - & 3.10 & - & 3.40 & - \\
\hline $\mathrm{Mn}$ & 0.03 & 0.02 & bd & bd & bd & 0.02 & 0.01 & 0.04 & - & 0.05 & - & 0.04 & - \\
\hline $\mathrm{Mg}$ & 1.45 & 1.52 & 1.06 & 1.14 & 1.07 & 1.11 & 0.25 & 1.18 & - & 1.30 & - & 1.33 & - \\
\hline $\mathrm{Ca}$ & bd & bd & bd & bd & bd & 0.01 & 0.02 & bd & - & bd & - & bd & - \\
\hline $\mathrm{Na}$ & 0.09 & 0.09 & 0.11 & 0.10 & 0.11 & 0.14 & 0.03 & 0.12 & - & 0.13 & - & 0.14 & - \\
\hline K & 1.87 & 1.85 & 1.77 & 1.67 & 1.71 & 1.73 & 0.13 & 1.79 & - & 1.88 & - & 1.93 & - \\
\hline $\mathrm{Cl}$ & na & na & 0.03 & 0.04 & 0.03 & bd & bd & 0.03 & - & 0.03 & - & 0.04 & - \\
\hline $\mathrm{Zn}$ & 0.02 & bd & na & na & na & 0.01 & 0.02 & 0.02 & - & 0.02 & - & 0.01 & - \\
\hline $\mathrm{Ba}$ & bd & bd & bd & bd & bd & 0.01 & 0.01 & bd & - & bd & - & bd & - \\
\hline sum & 15.47 & 15.46 & 15.25 & 15.27 & 15.31 & 15.33 & 0.21 & 16.12 & - & 16.76 & - & 17.50 & - \\
\hline $\mathrm{X}$ & 1.97 & 1.94 & 1.87 & 1.78 & 1.82 & 1.89 & 0.19 & 1.91 & - & 2.00 & - & 2.07 & - \\
\hline Y & 5.48 & 5.52 & 5.35 & 5.44 & 5.46 & 5.16 & - & 6.17 & - & 6.71 & - & 7.39 & - \\
\hline $\mathrm{MgV}$ & 0.34 & 0.36 & 0.28 & 0.28 & 0.27 & 0.29 & 0.62 & 0.27 & - & 0.29 & - & 0.28 & - \\
\hline
\end{tabular}

The first number is the crystal and the second one represents the core (1) or the rim (2). Comparison with the Macusani and Morococala mean biotite compositions from literature "AR" is the abbreviation for andalusite-bearing rhyolites in Morgan et al. (1998). X is K + Na + Ba and Y is AlVI+Mg + Fe + Mn + Ti. Abbreviations: "na" stands for "not analyzed" and "bd" stands for "below detection" 

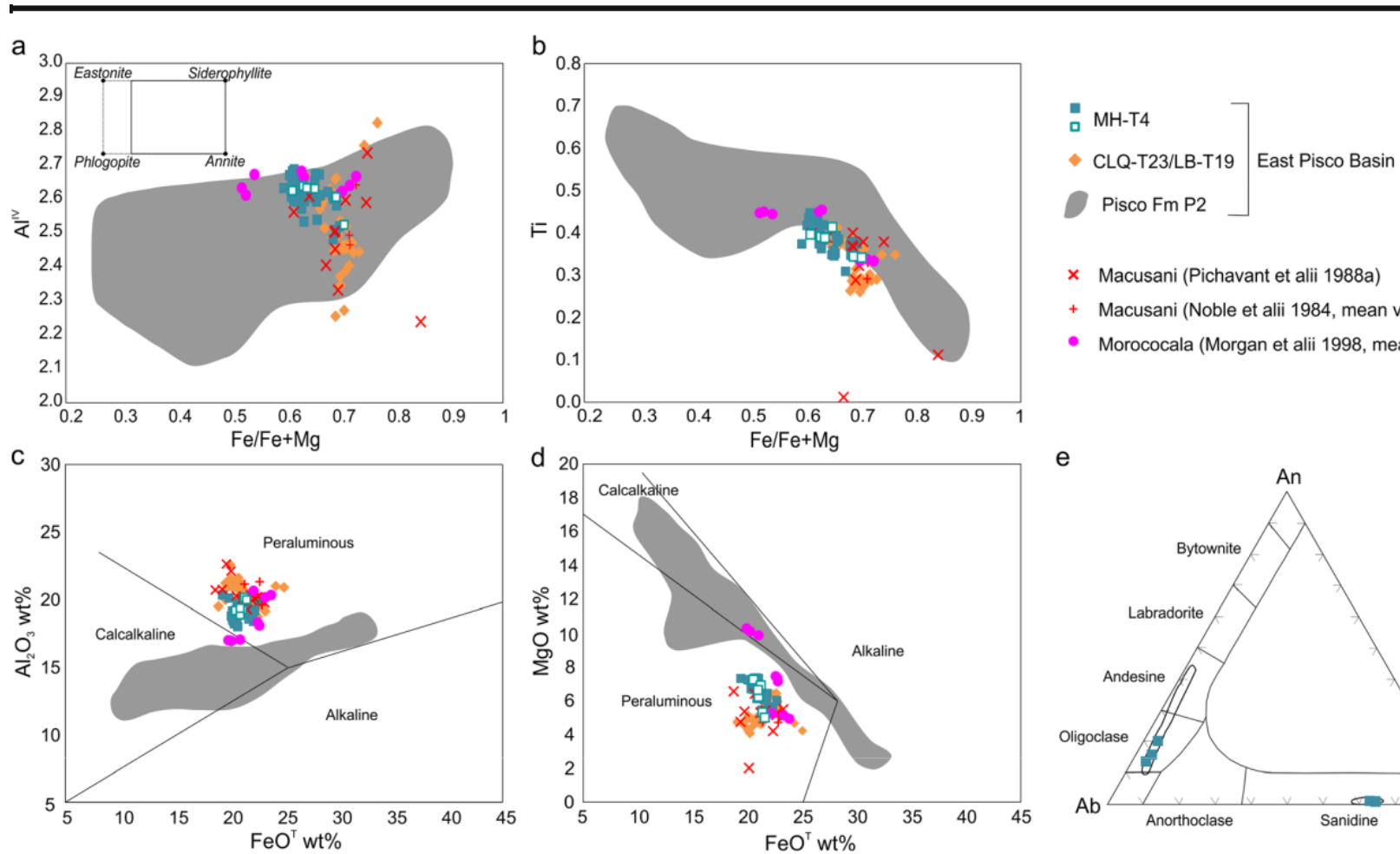

X Macusani (Pichavant et alii 1988a)

+ Macusani (Noble et alii 1984, mean values)

- Morococala (Morgan et alii 1998, mean values)

Fig. 5 Biotite compositional diagrams. a Classification of MH-T4 and CLQ-T23/LB-T19 biotite in the $\mathrm{Al}^{\mathrm{IV}}$ vs $\mathrm{Fe} / \mathrm{Fe}+\mathrm{Mg}$ diagram of Clarke (1981). For MH-T4, open symbols represent analyses at the Institut des Sciences de la Terre d'Orleans (Orléans, France), and close symbols represent analyses at the Università di Milano (Dipartimento di Scienze della Terra "Ardito Desio," Milano, Italy). Comparison with biotite composition from tephra of the P2 allomember (gray field) and from SP Macusani and Morococala volcanic fields. b MH-T4 and CLQ-T23/LB-

current) with counting time of $30 \mathrm{~s}$ on the elemental peak and $10 \mathrm{~s}$ on the background. The beam diameter was $3 \mu \mathrm{m}$ for phenocrysts and $10 \mu \mathrm{m}$ for glass shards, i.e., enlarged in order to avoid Na migration under the beam. EPMA analyses were also performed for major elements, plus zinc and fluorine, at the Institut des Sciences de la Terre d'Orleans (ISTO) (Orléans, France) facilities with a SX Five CAMECA instrument, with $15-\mathrm{kVaccelerating} \mathrm{voltage} \mathrm{and} \mathrm{5-nA} \mathrm{beam} \mathrm{current.}$ The counting time was $10 \mathrm{~s}$ on peak and $5 \mathrm{~s}$ on background; the spot size was defocused to $10 \mu \mathrm{m}$ on glasses and focused to $1-2 \mu \mathrm{m}$ on minerals.

Trace element concentrations in the MH-T4 matrix glass were analyzed by LA-ICP-MS (Laser AblationInductively Coupled Plasma-Mass Spectrometry) at Orléans (joint ISTO-IRAMAT facility). A RESOlution ArF excimer laser (LA; $\lambda=193 \mathrm{~nm}$ ) coupled to an Agilent TripleQuad ICPMS was used. Ablation was per-formed at a frequency of $10 \mathrm{~Hz}$ and an energy of

$2.5 \mathrm{~J} \mathrm{.cm}^{-2}$. A beam diameter of $10 \mu \mathrm{m}$ was used. NIST610 was used as external standard and obsidian glass JV2 (Pichavant et al. 1987) as secondary standard. Since small spot sizes were necessary, systematic analyses were performed on JV2 at different spot sizes between 100 and
$\mathrm{T} 19$ biotite in the $\mathrm{Ti}$ (apfu) vs $\mathrm{Fe} / \mathrm{Fe}+\mathrm{Mg}$ diagram. Symbols and field as in 5a. c Composition of MH-T4 and CLQ-T23/LB-T19 biotite in $\mathrm{Al}_{2} \mathrm{O}_{3}$ vs $\mathrm{FeO}^{\mathrm{T}}$ diagram according to Abdel-Rahman (1994). Symbols and field as in (a). d Composition of MH-T4 and CLQ-T23/LB-T19 biotite in $\mathrm{MgO}$ vs $\mathrm{FeO}^{\mathrm{T}}$ diagram according to Abdel-Rahman (1994). Symbols and field as in (a). e Chemical ternary diagram of feldspar phenocrysts from the MH-T4 tephra. Comparison with the SP Macusani feldspar fields

$10 \mu \mathrm{m}$ to check that decreasing spot sizes had no significant effect on element concentrations. A total of three successful analyses were obtained. The raw data were processed off-line using the Glitter software (Griffin 2008) where time-resolved signals were examined one by one.

For ${ }^{39} \mathrm{Ar}-{ }^{40} \mathrm{Ar}$ analysis, biotite and muscovite phenocrysts were carefully handpicked under a stereomicroscope, making particular care to avoid any contamination, following Bosio et al. (2020b). ${ }^{39} \mathrm{Ar}-{ }^{40} \mathrm{Ar}$ dating were performed with the NuInstruments ${ }^{\mathrm{TM}}$ Noblesse ${ }^{\circledR}$ noble gas mass spectrometer at the Università di Milano-Bicocca using the step-heating procedure described by Bosio et al. (2020b).

\section{Results}

\section{Field and petrographic description}

The sample MH-T4 was collected from an ash layer outcrop-ping at Cerros la Mama y la Hija (Figs. 2, 3), at the base of the P2 allomember, $3 \mathrm{~m}$ above the PE0.2 unconformity (Fig. 3). The layer is a 5 -cm-thick white ash with visible fine-grained 
Table 3 EPMA analyses on muscovite phenocrysts of MH-T4 tephra

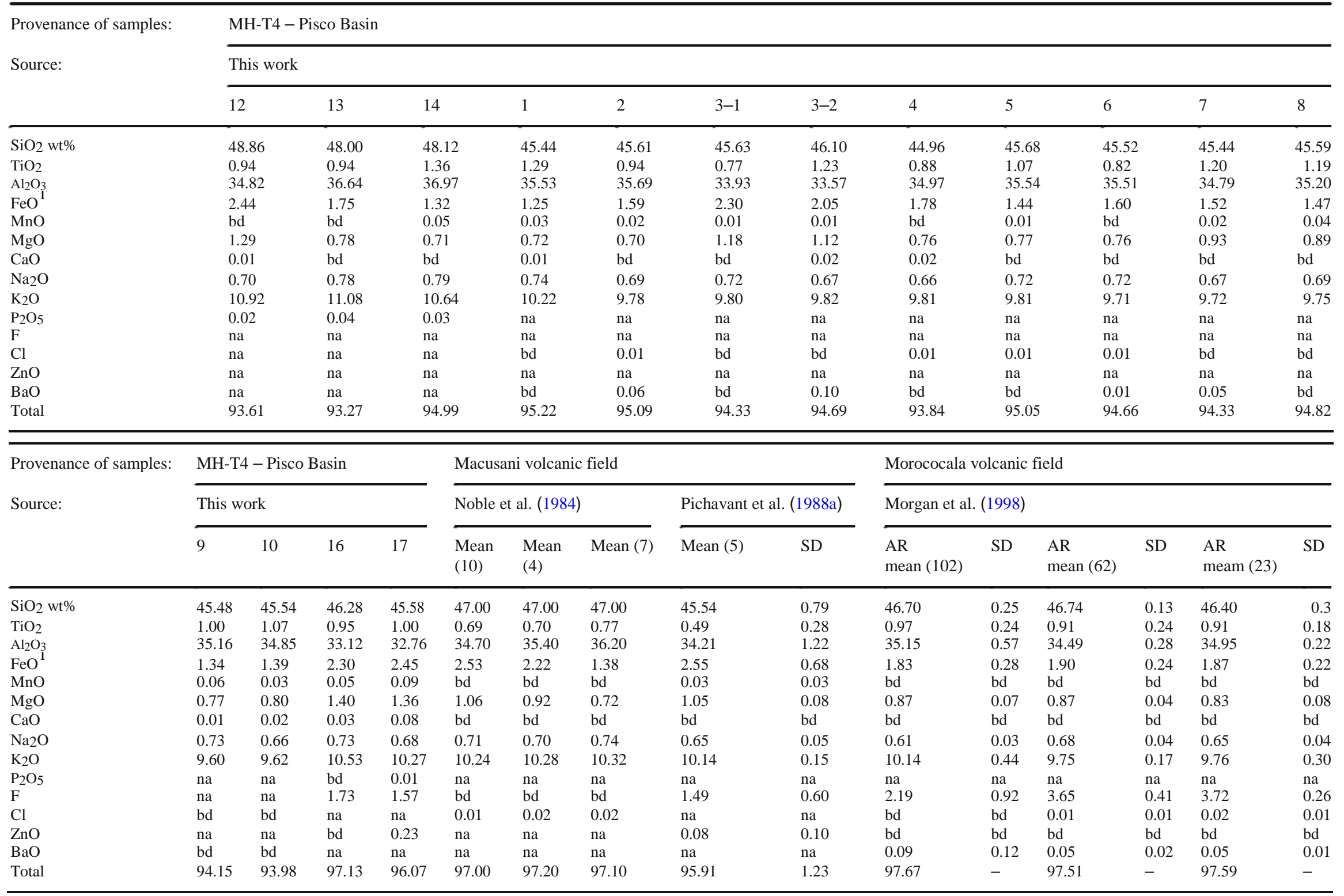

Comparison with the Macusani and Morococala mean muscovite compositions from literature. "AR" is the abbreviation for andalusite-bearing rhyolites in Morgan et al. (1998). Abbreviations;

"na" stands for "not analyzed" and "bd" stands for "below detection" 
Table 4 EPMA analyses on feldspar phenocrysts of MH-T4 tephra

\begin{tabular}{|c|c|c|c|c|c|c|c|c|c|c|c|c|c|c|c|c|}
\hline \multirow{4}{*}{$\begin{array}{l}\text { Provenance of } \\
\text { samples: } \\
\text { Source: } \\
\text { Type: }\end{array}$} & \multicolumn{6}{|c|}{ MH-T4 - Pisco Basin } & \multirow{2}{*}{\multicolumn{4}{|c|}{$\frac{\text { Macusani volcanic field }}{\text { Pichavant et al. (1988a) }}$}} & \multicolumn{6}{|c|}{ Morococala volcanic field } \\
\hline & \multicolumn{6}{|c|}{ This work } & & & & & \multicolumn{6}{|c|}{ Morgan et al. (1998) } \\
\hline & \multicolumn{3}{|c|}{ Sanidine } & \multicolumn{3}{|c|}{ Plagioclase } & \multicolumn{2}{|c|}{ Sanidine } & \multicolumn{2}{|c|}{ Plagioclase } & \multicolumn{4}{|c|}{ Plagioclase } & \multicolumn{2}{|l|}{ Sanidine } \\
\hline & 12 & 13 & 15 & 16 & 19 & 20 & $\begin{array}{l}\text { Mean } \\
(3)\end{array}$ & $\mathrm{SD}$ & $\begin{array}{l}\text { Mean } \\
(7)\end{array}$ & SD & $\begin{array}{l}\text { AR mean } \\
(282)\end{array}$ & SD & $\begin{array}{l}\text { AR mean } \\
(288)\end{array}$ & SD & $\begin{array}{l}\text { AR mean } \\
(537)\end{array}$ & $\mathrm{SD}$ \\
\hline $\mathrm{SiO}_{2} \mathrm{wt} \%$ & 64.44 & 59.60 & 64.36 & 61.67 & 63.18 & 62.55 & 65.06 & 0.27 & 62.34 & 3.12 & 62.89 & 1.46 & 63.53 & 1.05 & 64.50 & 0.32 \\
\hline $\mathrm{Al}_{2} \mathrm{O}_{3}$ & 19.21 & 18.25 & 19.21 & 23.54 & 22.26 & 22.63 & 18.90 & 0.06 & 23.56 & 1.94 & 23.12 & 1.03 & 22.72 & 0.66 & 19.33 & 0.11 \\
\hline $\mathrm{FeO}^{\mathrm{T}}$ & 0.06 & 0.03 & 0.01 & 0.06 & 0.08 & bd & na & na & na & na & 0.02 & 0.01 & 0.05 & 0.03 & 0.02 & 0.01 \\
\hline $\mathrm{CaO}$ & 0.05 & 0.07 & 0.10 & 4.11 & 2.85 & 3.29 & bd & bd & 4.58 & 2.58 & 3.58 & 1.15 & 3.13 & 0.81 & 0.05 & 0.02 \\
\hline $\mathrm{Na}_{2} \mathrm{O}$ & 2.67 & 2.81 & 2.65 & 8.51 & 9.41 & 9.15 & 3.40 & 0.13 & 8.42 & 1.40 & 8.89 & 0.62 & 9.14 & 0.47 & 3.05 & 0.23 \\
\hline $\mathrm{K}_{2} \mathrm{O}$ & 12.03 & 11.54 & 11.87 & 0.68 & 0.68 & 0.77 & 11.60 & 0.24 & 0.63 & 0.23 & 0.67 & 0.12 & 0.71 & 0.06 & 12.07 & 0.28 \\
\hline $\mathrm{BaO}$ & 0.22 & 0.38 & 0.13 & $\mathrm{bd}$ & bd & 0.01 & 0.07 & 0.08 & na & na & na & na & na & na & 0.12 & 0.06 \\
\hline An & 0.26 & 0.37 & 0.52 & 20.23 & 13.78 & 15.84 & 0.00 & 0.00 & 22.38 & 12.90 & 17.49 & 5.70 & 15.26 & 4.00 & 0.30 & 0.10 \\
\hline $\mathrm{Ab}$ & 25.05 & 26.71 & 25.14 & 75.80 & 82.33 & 79.71 & 30.78 & 1.29 & 73.96 & 11.67 & 78.61 & 5.20 & 80.62 & 3.80 & 27.62 & 2.00 \\
\hline Or & 74.69 & 72.92 & 74.34 & 3.97 & 3.89 & 4.45 & 69.22 & 1.29 & 3.66 & 1.31 & 3.90 & 0.70 & 4.12 & 0.40 & 71.90 & 1.90 \\
\hline Total & 98.72 & 92.69 & 98.36 & 98.57 & 98.50 & 98.46 & 99.00 & 0.36 & 99.54 & 1.04 & 99.32 & - & 99.43 & - & 99.35 & - \\
\hline
\end{tabular}

Plagioclase and sanidine phenocrysts are compared with the Macusani and Morococala mean compositions from literature. "AR" is the abbreviation for andalusite-bearing rhyolites in Morgan et al. (1998). Abbreviations; "na" stands for "not analyzed" and "bd" stands for "below detection"

biotite, which can be traced laterally for several tens of meters in sandstones. The CLQ-T23 and LB-T19 ash layers were sampled at Cerro los Quesos and Cerro la Bruja localities, re-spectively (Fig. 2). These ash layers were described and correlat-ed based on stratigraphy, grain-size, shard morphology, and glass and biotite composition by Bosio et al. (2019), and they will be referred to as CLQ-T23/LB-T19 tephra herein after.

Under the optical microscope, the MH-T4 ash consists of highly vesiculated, colorless glass shards and of loose crystals of juvenile biotite, feldspars, and minor

Table 5 EPMA analyses on $\mathrm{Al}_{2} \mathrm{SiO}_{5}$ minerals of MH-T4 tephra

\begin{tabular}{|c|c|c|c|c|c|c|c|c|c|c|c|c|c|c|c|c|}
\hline \multirow{4}{*}{$\begin{array}{l}\text { Provenance of } \\
\text { samples: } \\
\text { Source: } \\
\text { Type: }\end{array}$} & \multicolumn{6}{|c|}{ MH-T4 - Pisco Basin } & \multicolumn{6}{|c|}{ Macusani volcanic field } & \multirow{2}{*}{\multicolumn{4}{|c|}{$\frac{\text { Morococala volcanic field }}{\text { Morgan et al. (1998) }}$}} \\
\hline & \multicolumn{6}{|c|}{ This work } & \multicolumn{2}{|c|}{ Noble et al. (1984) } & \multicolumn{4}{|c|}{ Pichavant et al. (1988a) } & & & & \\
\hline & \multicolumn{6}{|c|}{$\mathrm{Al}_{2} \mathrm{SiO}_{5}$ mineral } & \multirow{2}{*}{$\frac{\text { Sillimanite }}{\text { Range }}$} & \multirow{2}{*}{$\frac{\text { Andalusite }}{\text { Range }}$} & \multicolumn{2}{|c|}{ Sillimanite } & \multicolumn{2}{|c|}{ Andalusite } & \multicolumn{2}{|c|}{ Andalusite } & \multicolumn{2}{|c|}{ Andalusite } \\
\hline & 11 & 12 & 14 & 17 & 18 & 21 & & & $\begin{array}{l}\text { Mean } \\
(2)\end{array}$ & $\mathrm{SD}$ & $\begin{array}{l}\text { Mean } \\
\text { (3) }\end{array}$ & SD & $\begin{array}{l}\text { AR } \\
\text { mean } \\
(34)\end{array}$ & $\mathrm{SD}$ & $\begin{array}{l}\text { AR } \\
\text { mean } \\
(39)\end{array}$ & $\mathrm{SD}$ \\
\hline $\mathrm{SiO}_{2} \mathrm{wt} \%$ & 38.25 & 36.16 & 36.20 & 35.60 & 36.88 & 36.07 & na & na & 36.40 & 0.37 & 37.25 & 0.80 & 36.84 & 0.19 & 36.99 & 0.07 \\
\hline $\mathrm{TiO}_{2}$ & bd & bd & 0.01 & 0.06 & bd & bd & $0.01-0.11$ & $0.01-0.34$ & 0.03 & 0.04 & 0.04 & 0.04 & 0.10 & 0.03 & bd & bd \\
\hline $\mathrm{Al}_{2} \mathrm{O}_{3}$ & 61.51 & 63.86 & 64.37 & 64.51 & 62.75 & 64.36 & na & na & 63.33 & 0.27 & 62.14 & 0.34 & 62.02 & 0.36 & 62.53 & 0.04 \\
\hline $\mathrm{FeO}^{\mathrm{T}}$ & 0.22 & 0.26 & 0.08 & 0.22 & 0.13 & 0.18 & $0.04-0.44$ & $0.17-0.80$ & 0.18 & 0.05 & 0.24 & 0.05 & 0.61 & 0.25 & 0.29 & 0.02 \\
\hline $\mathrm{MnO}$ & bd & 0.03 & 0.04 & 0.01 & bd & 0.04 & na & na & 0.05 & 0.07 & 0.03 & 0.03 & bd & bd & bd & bd \\
\hline $\mathrm{MgO}$ & 0.02 & bd & bd & bd & 0.02 & bd & na & na & 0.02 & 0.03 & 0.04 & 0.02 & 0.05 & 0.03 & bd & bd \\
\hline $\mathrm{CaO}$ & $\mathrm{bd}$ & 0.03 & bd & 0.02 & bd & bd & $0.01-0.20$ & bd & 0.02 & 0.03 & 0.01 & 0.01 & bd & $\mathrm{bd}$ & bd & bd \\
\hline $\mathrm{Na}_{2} \mathrm{O}$ & bd & 0.03 & bd & bd & 0.02 & bd & na & na & bd & bd & bd & bd & bd & bd & bd & bd \\
\hline $\mathrm{K}_{2} \mathrm{O}$ & bd & 0.03 & bd & 0.01 & 0.09 & bd & na & na & bd & bd & bd & bd & bd & bd & bd & bd \\
\hline Total & 98.73 & 100.41 & 100.72 & 100.42 & 99.90 & 100.67 & - & - & 100.02 & 0.06 & 99.75 & 1.01 & 99.62 & - & 99.81 & - \\
\hline
\end{tabular}

Comparison with the Macusani and Morococala mean compositions of sillimanite and andalusite from literature. "AR" is the abbreviation for andalusite-bearing rhyolites in Morgan et al. (1998). Abbreviations; "na" stands for "not analyzed" and "bd" stands for "below detection" 
Table 6 EPMA analyses on distal ash glass shards of MH-T4 tephra reported as means and standard deviations

\begin{tabular}{|c|c|c|c|c|c|c|c|c|c|c|c|c|c|}
\hline \multirow{4}{*}{$\begin{array}{l}\text { Provenance of } \\
\text { samples: } \\
\text { Source: } \\
\text { Type: }\end{array}$} & \multicolumn{8}{|c|}{$\begin{array}{l}\text { MH-T4 - Pisco Macusani volcanic field } \\
\text { Basin }\end{array}$} & \multicolumn{5}{|c|}{ Morococala volcanic field } \\
\hline & \multicolumn{2}{|c|}{ This work } & \multirow{3}{*}{$\begin{array}{l}\text { London et al. } \\
\text { (1988) } \\
\text { Homogeneous } \\
\text { aphyric obsidian }\end{array}$} & \multicolumn{5}{|c|}{ Pichavant et al. (1987) } & \multicolumn{5}{|c|}{ Morgan et al. (1998) } \\
\hline & \multicolumn{2}{|c|}{$\begin{array}{l}\text { Distal ashes } \\
\text { glass shards }\end{array}$} & & \multicolumn{5}{|c|}{ Glass pebbles } & \multicolumn{5}{|c|}{$\mathrm{CR}$ matrix glass } \\
\hline & $\begin{array}{l}\text { Mean } \\
(15)\end{array}$ & $\mathrm{SD}$ & & $\begin{array}{l}\text { JV1 mean } \\
\text { (5) }\end{array}$ & $\begin{array}{l}\mathrm{CC} 2 \text { mean } \\
(2)\end{array}$ & $\begin{array}{l}\text { CM1 mean } \\
(2)\end{array}$ & $\begin{array}{l}\text { CM2 mean } \\
(2)\end{array}$ & $\begin{array}{l}\mathrm{CM} 2 \text { red } \\
\text { zone }\end{array}$ & $\begin{array}{l}14 \text { mean } \\
(136)\end{array}$ & $\begin{array}{l}23 \text { mean } \\
(55)\end{array}$ & $\begin{array}{l}24 \text { mean } \\
(30)\end{array}$ & $\begin{array}{l}25 \text { mean } \\
(17)\end{array}$ & $\begin{array}{l}28 \text { mean } \\
(13)\end{array}$ \\
\hline $\mathrm{SiO}_{2} \mathrm{wt} \%$ & 73.28 & 2.55 & 72.32 & 72.26 & 72.69 & 72.29 & 72.31 & 71.95 & 73.75 & 74.08 & 73.78 & 74.13 & 74.11 \\
\hline $\mathrm{TiO}_{2}$ & 0.05 & 0.03 & 0.02 & 0.07 & bd & 0.02 & 0.04 & bd & 0.07 & 0.07 & 0.07 & 0.07 & 0.07 \\
\hline $\mathrm{Al}_{2} \mathrm{O}_{3}$ & 14.24 & 0.50 & 15.63 & 15.79 & 16.00 & 15.92 & 15.75 & 15.62 & 14.07 & 14.30 & 14.01 & 14.12 & 14.28 \\
\hline $\mathrm{FeO}^{\mathrm{T}}$ & 0.68 & 0.11 & 0.52 & 0.54 & 0.56 & 0.59 & 0.47 & 2.75 & 0.80 & 0.81 & 0.78 & 0.74 & 0.68 \\
\hline $\mathrm{MnO}$ & 0.05 & 0.03 & 0.06 & 0.03 & 0.03 & 0.08 & 0.04 & 0.12 & 0.04 & 0.03 & 0.03 & 0.03 & 0.04 \\
\hline $\mathrm{MgO}$ & 0.08 & 0.03 & 0.02 & 0.02 & 0.01 & 0.02 & 0.01 & 0.03 & 0.08 & 0.08 & 0.08 & 0.08 & 0.08 \\
\hline $\mathrm{CaO}$ & 0.44 & 0.07 & 0.23 & 0.19 & 0.10 & 0.19 & 0.15 & 0.26 & 0.34 & 0.40 & 0.46 & 0.38 & 0.39 \\
\hline $\mathrm{Na} 2 \mathrm{O}$ & 3.57 & 0.17 & 4.10 & 4.29 & 4.30 & 4.32 & 4.27 & 3.93 & 2.96 & 3.19 & 3.79 & 3.09 & 2.77 \\
\hline $\mathrm{K}_{2} \mathrm{O}$ & 4.95 & 0.18 & 3.53 & 3.83 & 3.77 & 3.74 & 3.88 & 3.56 & 5.19 & 4.98 & 4.63 & 4.92 & 5.31 \\
\hline $\mathrm{P}_{2} \mathrm{O}_{5}$ & 0.27 & 0.09 & 0.58 & na & na & na & na & na & 0.25 & 0.24 & 0.30 & 0.23 & 0.24 \\
\hline $\mathrm{ZnO}$ & 0.01 & 0.01 & na & na & na & na & na & na & na & na & na & na & na \\
\hline F & 0.27 & 0.03 & 1.30 & 1.34 & 1.27 & 1.35 & 1.33 & 1.27 & 0.36 & 0.24 & 0.39 & 0.26 & 0.31 \\
\hline $\mathrm{Cl}$ & bd & - & 0.05 & na & na & na & na & na & 0.11 & 0.11 & 0.11 & 0.12 & 0.14 \\
\hline $\mathrm{Na}_{2} \mathrm{O}+\mathrm{K}_{2} \mathrm{O}$ & 8.52 & 0.24 & 7.63 & 8.12 & 8.07 & 8.06 & 8.15 & 7.49 & 8.15 & 8.17 & 8.42 & 8.01 & 8.08 \\
\hline $\mathrm{A} / \mathrm{CNK}$ & 1.18 & 0.02 & 1.42 & 1.37 & 1.41 & 1.38 & 1.37 & 1.45 & 1.27 & 1.26 & 1.16 & 1.27 & 1.30 \\
\hline Total & 92.76 & 1.22 & 99.78 & 98.35 & 98.73 & 98.52 & 98.25 & 99.49 & 97.87 & 98.41 & 98.26 & 98.03 & 98.26 \\
\hline
\end{tabular}

Comparison with the Macusani and Morococala matrix glass analyses from literature. "CR" is the abbreviation for cordierite-bearing rhyolites in Morgan et al. (1998). Concentrations are normalized to 100 and the original EPMA total of MH-T4 is shown. Abbreviations: "na" stands for "not analyzed" and "bd" stands for "below detection" 

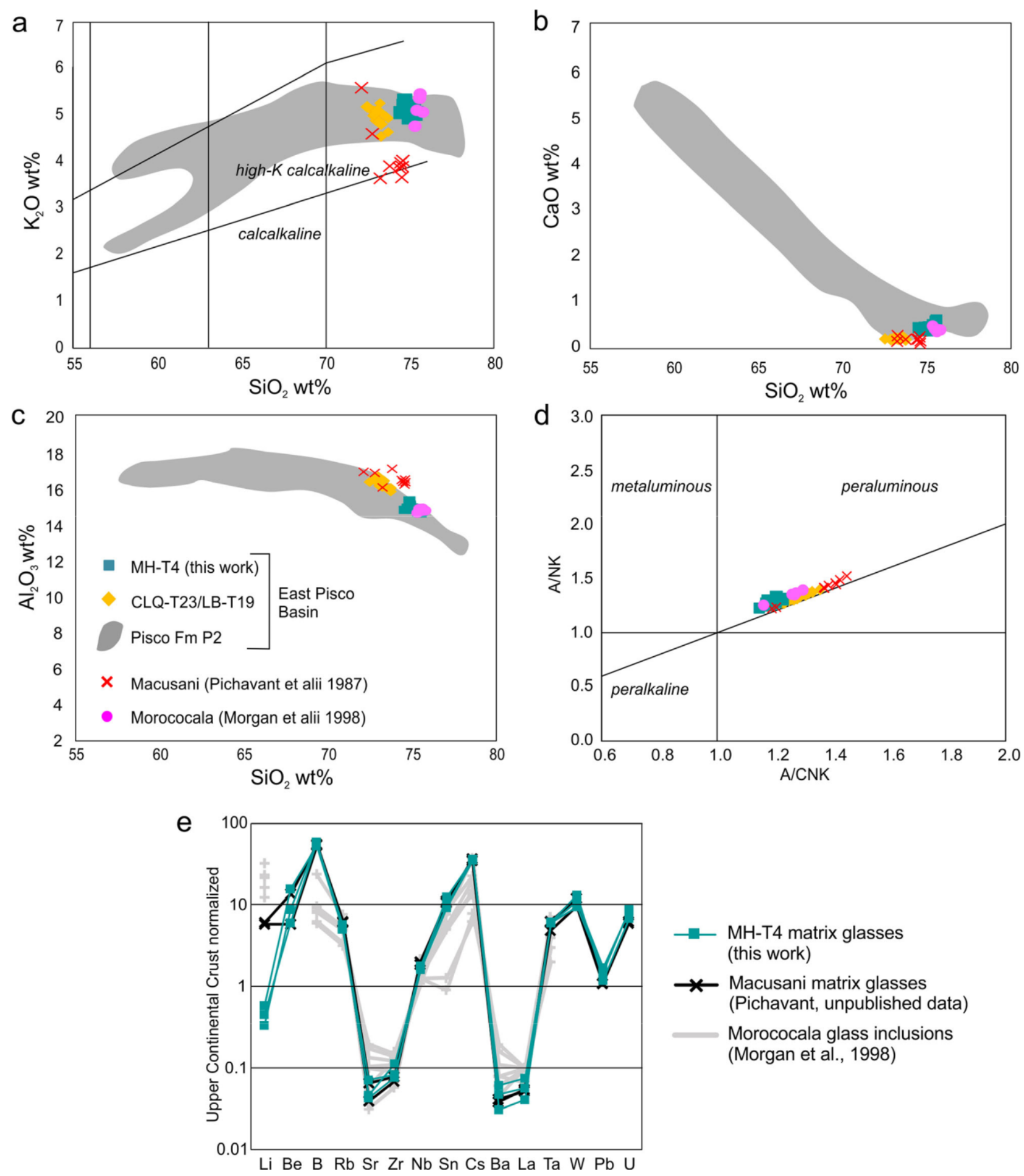

Fig. 6 Composition of matrix glass. a Harker $\mathrm{K}_{2} \mathrm{O}$ vs $\mathrm{SiO}_{2}$ diagram of glass shards showing the glass composition of MH-T4 and CLQ-T23/LB-T19 tephra. Comparison with glass composition from tephra of the $\mathrm{P} 2$ allomember (gray field) and from SP Macusani and Morococala volcanic fields. Fields from Peccerillo and Taylor (1976). b $\mathrm{CaO}$ vs $\mathrm{SiO}_{2}$ diagram of glass shards showing the glass composition of MH-T4 and CLQ-T23/LB-T19 tephra. Comparison with glass composition from tephra of the P2 allomember (gray field) and from SP Macusani and Morococala volcanic fields. c $\mathrm{Al}_{2} \mathrm{O}_{3}$ vs $\mathrm{SiO}_{2}$ diagram of glass shards showing the glass composition of MH-T4 and CLQT23/LB-T19 tephra. Comparison with glass composition from tephra of the P2 allomember (gray field) and from SP Macusani and Morococala volcanic fields. d A/NK vs A/CNK diagram following Shand (1943) of glass shards showing the glass composition of $\mathrm{MH}-$ T4 and CLQ-T23/LB-T19 tephra. Comparison with glass composition from SP Macusani and Morococala volcanic fields. e Trace element LA-ICP-MS analysis of matrix glasses for MH-T4 (this work), matrix glasses for Macusani (M. Pichavant, unpublished data), and glass inclusions in quartz for Morococala (Morgan et al. 1998). Values are normalized to upper continental crust (normalizing values from Taylor and McLennan 1985) 
Table 7 Trace element LA-ICP-MS analysis of matrix glasses for MH-T4 tephra

\begin{tabular}{llll}
\hline & MH-T4 glass 1 & MH-T4 glass 2 & MH-T4 glass 3 \\
\hline $\mathrm{Li}(\mathrm{ppm})$ & 9.04 & 6.70 & $<11.62$ \\
$\mathrm{Be}$ & 18.2 & 26.3 & 47.5 \\
$\mathrm{~B}$ & 865 & 873 & 803 \\
$\mathrm{Rb}$ & 645 & 584 & 558 \\
$\mathrm{Sr}$ & 15.1 & 16.2 & 25.1 \\
$\mathrm{Zr}$ & 14.65 & 21.2 & 15.96 \\
$\mathrm{Nb}$ & 21.0 & 21.6 & 19.24 \\
$\mathrm{Sn}$ & 55.1 & 68.8 & 51.2 \\
$\mathrm{Cs}$ & 166 & 167 & 162 \\
$\mathrm{Ba}$ & 17.08 & 26.0 & 33.5 \\
$\mathrm{La}$ & 1.24 & 1.71 & 2.25 \\
$\mathrm{Ta}$ & 6.12 & 6.02 & 5.96 \\
$\mathrm{~W}$ & 26.2 & 19.1 & 22.0 \\
$\mathrm{~Pb}$ & 20.3 & 25.7 & 29.1 \\
$\mathrm{U}$ & 19.3 & 24.9 & 23.4 \\
\hline
\end{tabular}

muscovite. An $\mathrm{Al}_{2} \mathrm{SiO}_{5}$ mineral phase, andalusite or sillimanite, was also detected inspecting the sample with SEM-EDS (Fig. 4). Biotite, muscovite, feldspar, and andalusite/sillimanite are coated by juvenile rhyolitic glass (Fig. 4). The CLQ-T23/LB-T19 tephra consists of colorless glass shards with a bubble-wall morphology and only a very few small phenocrysts of biotite. Through grain-size analysis, we find that both tephra samples are unimodal and poorly sorted (see Fig. 4e), and they are composed by extremely fine ash $(<1 / 16 \mathrm{~mm})$ following the classification of White and Houghton (2006).

\section{Mineral and glass composition}

Biotite phenocrysts of both tephra layers (MH-T4 and CLQT23/LB-T19) show a rather homogeneous major element composition from core to rim (Tables 1 and 2, and Bosio et al. 2019). From the point of view of the biotite classification in an $\mathrm{Al}^{\mathrm{IV}}$ vs $\mathrm{Fe} / \mathrm{Fe}+\mathrm{Mg}$ diagram, they are on the siderophyllite-rich side of the field representing the composition of biotite found in the P2 allomember tephra (Fig. 5a). Biotite compo-sition, for both tephra layers, can be distinguished from the rest of the P2 tephra in $\mathrm{Ti}$ vs $\mathrm{Fe} / \mathrm{Fe}+$ $\mathrm{Mg}$ (Fig. 5b). Moreover, biotite phenocrysts are richer in $\mathrm{Al}_{2} \mathrm{O}_{3}$ wt $\%$ with respect to the rest of P2 tephra (Fig. 5c) and fall within the field of the micas found in peraluminous granite in the discrimination diagrams of Abdel-Rahman (1994) (Fig. 5c, d). They show detectable $\mathrm{ZnO}$ contents, while $\mathrm{F}$ is mostly below detection (Tables 1 and 2).
The peculiarity of the MH-T4 tephra is the presence of muscovite crystals, which are juvenile phenocrysts as indicated by the coating of rhyolitic glass (Fig. 4c). Muscovite contains significant amounts of $\mathrm{Ti}, \mathrm{Fe}$, and $\mathrm{Mg}$, detectable $\mathrm{ZnO}$, and high F, similarly to muscovite in SP rocks (Table 3). Feldspar phenocrysts coated by rhyolitic glass are both plagioclase (oligoclase) and sanidine (Table 4, Fig. 5e). MH-T4 tephra shows, also, the presence of $\mathrm{Al}_{2} \mathrm{SiO}_{5}$ minerals in rhyolitic glass, probably andalusite or sillimanite (Table 5).

The composition of the matrix glass is rhyolitic and, on the basis of $\mathrm{K}_{2} \mathrm{O}$ vs $\mathrm{SiO}_{2}$ diagram, corresponds to magmas belonging to the high-K calc-alkaline series (Table 6, Fig. 6a). When normalized to 100 , the silica and $\mathrm{CaO}$ content is slightly higher and the $\mathrm{Al}_{2} \mathrm{O}_{3}$ is slightly lower for MH-T4 than for CLQ-T23/LB-T19 (Fig. 6b, c). The low microprobe analysis totals may indicate a high concentration of magmatic water or that hydration of glass occurred following the subaqueous deposition of ash. Since the removal of mobile cations follow-ing interaction with an aqueous fluid may cause an increase of the $\mathrm{A} / \mathrm{CNK}$ (molar $\mathrm{Al}_{2} \mathrm{O}_{3} / \mathrm{CaO}+\mathrm{Na}_{2} \mathrm{O}+$ $\mathrm{K}_{2} \mathrm{O}$ ) ratio of glass, the analyses showing at the same time low $\mathrm{K}_{2} \mathrm{O}$, low micro-probe total, and high $\mathrm{A} / \mathrm{CNK}$ were discarded. The obtained A/CNK ratio of (mean value) 1.18 in MH-T4 and 1.28 in CLQ-T23/LB-T19 indicates a peraluminous composition (Fig. 6d).

The concentration of fluorine and of several trace elements was determined for MH-T4 matrix glasses. Fluorine is rather high (mean value of $2700 \mathrm{ppm}$, Table 6). The trace element composition of the matrix glass normalized to upper continental crust exhibits high $\mathrm{B}, \mathrm{Be}, \mathrm{Rb}, \mathrm{Cs}$, and $\mathrm{Sn}$ and low $\mathrm{Li}, \mathrm{Zr}$, $\mathrm{Sr}, \mathrm{Ba}$, and La (Table 7, Fig. 6e).

\section{${ }^{39} \mathrm{Ar}-{ }^{40} \mathrm{Ar}$ dating of the two-mica tephra}

The composition of MH-T4 biotite indicates a relatively high alkali site occupancy from 1.83 to 1.95 apfu, i.e., it is only slightly altered, which makes it suitable for ${ }^{39} \mathrm{Ar}-$ ${ }^{40} \mathrm{Ar}$ dating. The $\mathrm{K}$ concentration calculated from the ${ }^{39} \mathrm{Ar}$ release is substoichiometric. As discussed in more detail by Bosio et al. (2020b), slight alteration affects micas from marine tephra.

The age spectrum of MH-T4 biotite shows a clear plateau (Fig. 7a). We consider only the isochemical steps (sensu Villa et al. 2006), i.e., steps 2-6 having $\mathrm{Ca} / \mathrm{K}<$ 0.005 and/or steps $3-6$ with $\mathrm{Ca} / \mathrm{K}<0.003$ (see Fig. 7b). Both choices yield isochrons with an atmospheric intercept and a slightly elevated MSWD $=3$. The weighted average age of steps $2-6$ is $7.98 \pm 0.03 \mathrm{Ma}$.

The muscovite phenocrysts show a high alkali site occupancy, between 1.83 and 1.99 apfu, reflecting a very slight alteration. The age spectrum of MH-T4 mus-covite shows a plateau around 8 Ma (Fig. 7a). We chose the isochemical steps $3-9$ with $\mathrm{Ca} / \mathrm{K}<0.005$ and 


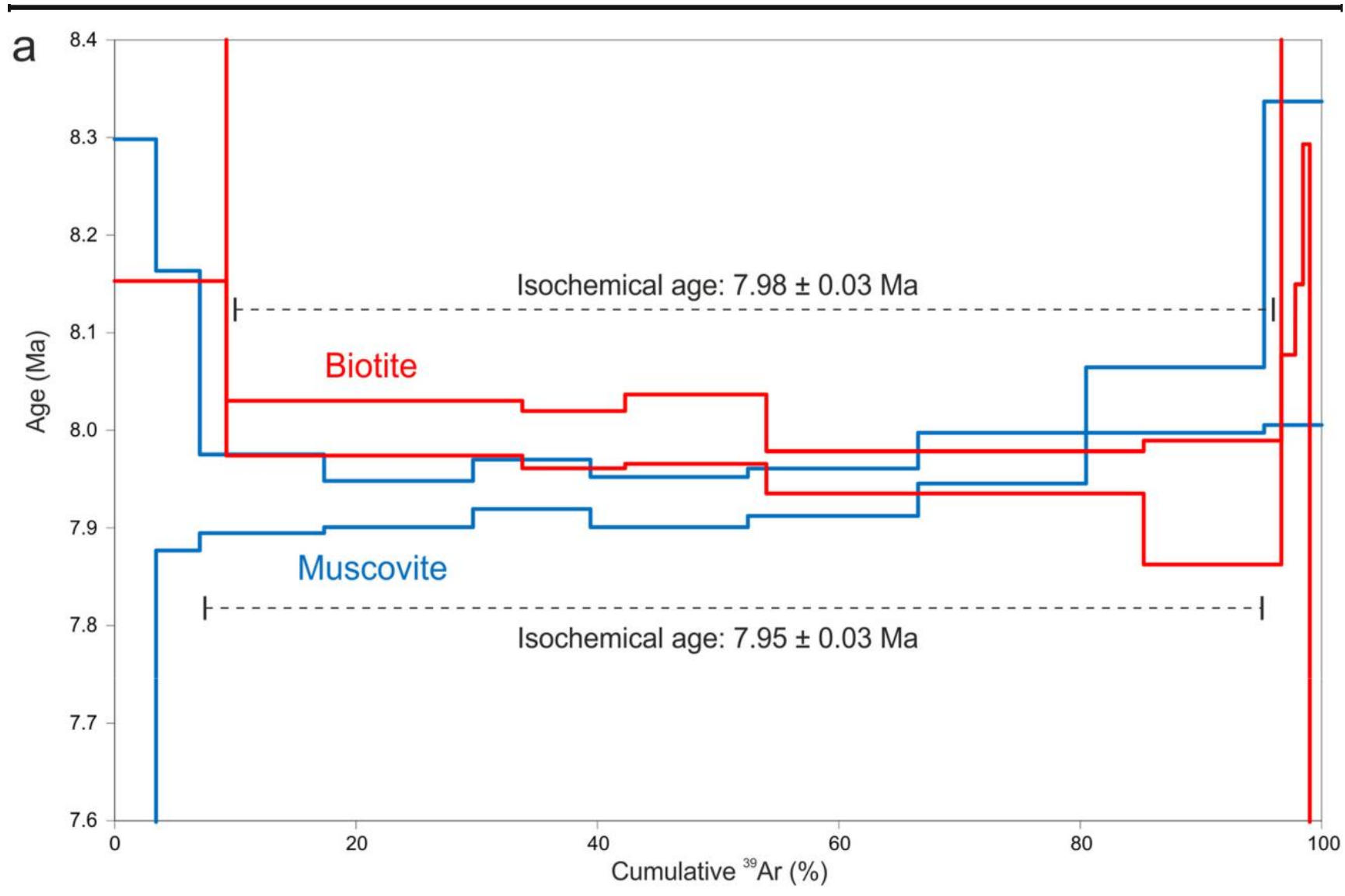

b

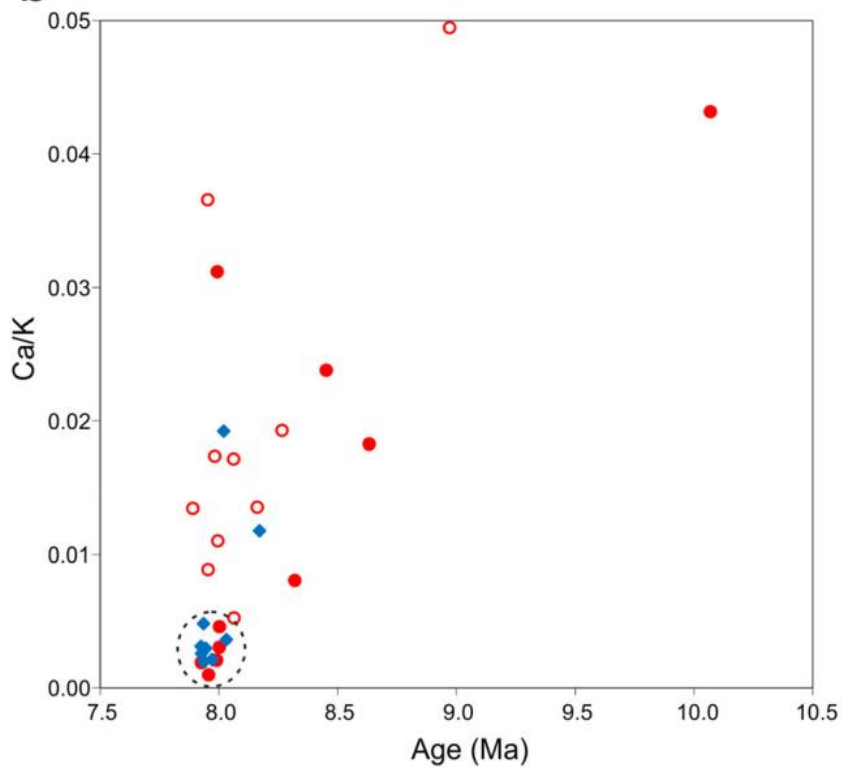

Fig. 7 Age spectra, $\mathrm{Ca} / \mathrm{K}$ vs age and $\mathrm{Cl} / \mathrm{K}$ vs age diagrams. a MH-T4 age spectra of biotite (red) and muscovite (blue) separates. Uncertainties are shown as $2 \sigma$. The isochemical ages (i.e., weighted average age of isochemical steps) are $7.98 \pm 0.03 \mathrm{Ma}$ for biotite and $7.95 \pm 0.03 \mathrm{Ma}$ for muscovite. b MH-T4 $\mathrm{Ca} / \mathrm{K}$ vs age diagram of biotite (red full circles), previous dated biotite (red empty circles) and muscovite (blue diamonds)
C

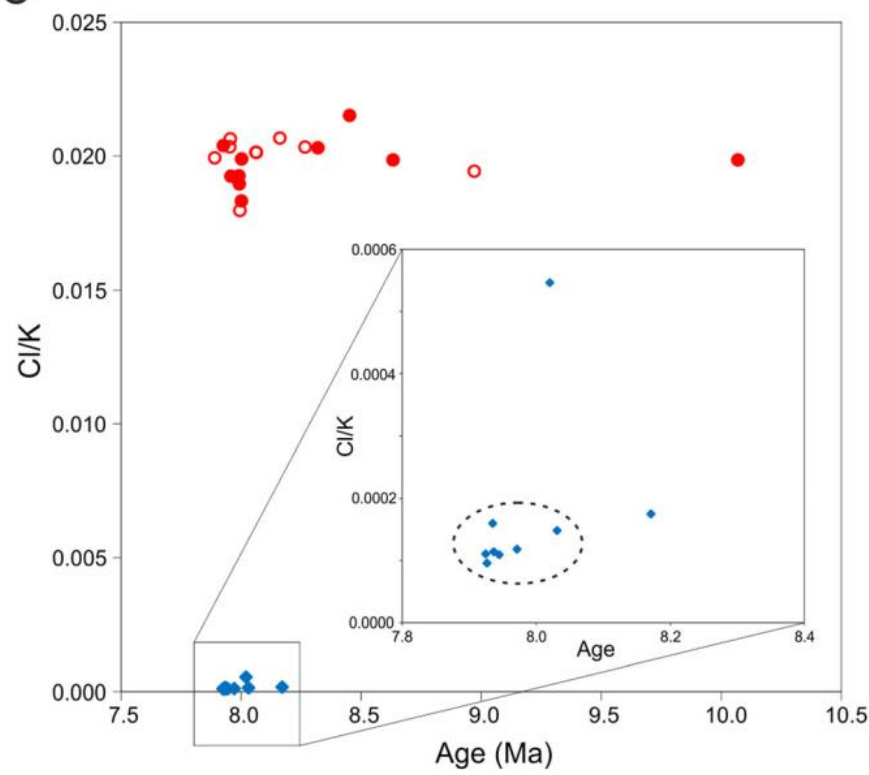

separates. The dashed ellipse includes the lowest $\mathrm{Ca} / \mathrm{K}$ steps considered for the age calculation. Uncertainties are smaller than symbols. C MH-T4 $\mathrm{Cl} / \mathrm{K}$ vs age diagram of biotite (red full circles) and previous dated biotite (red empty circles) with a close-up on the muscovite (blue diamonds) points. The dashed ellipse includes the lowest $\mathrm{Cl} / \mathrm{K}$ steps considered for the age calculation. Uncertainties are smaller than symbols 
Table $8{ }^{39} \mathrm{Ar}-{ }^{40} \mathrm{Ar}$ dating results of MH-T4 biotite phenocrysts

\section{MH-T4 biotite}

Weight $=0.01729 \mathrm{~g} ; \mathrm{J}=0.001008 ;[\mathrm{K}]=8.53 \% ;[\mathrm{Ca}]=440 \mathrm{ppm} ;[\mathrm{Cl}]=1680 \mathrm{ppm} ;$ Err. $=$ Error

\begin{tabular}{|c|c|c|c|c|c|c|c|c|c|c|}
\hline Step & 1 & 2 & 3 & 4 & 5 & 6 & 7 & 8 & 9 & 10 \\
\hline \multirow{4}{*}{$\begin{array}{l}\mathrm{T}\left({ }^{\circ} \mathrm{C}\right) \\
{ }^{40} \mathrm{Ar} \text { total } \\
{ }^{\mathrm{Err} .}{ }^{40} \mathrm{Ar} \\
{ }^{40} \mathrm{Ar} *\end{array}$} & 708 & 796 & 840 & 980 & 1033 & 1069 & 1098 & 1118 & 1201 & 1286 \\
\hline & $3.15 \times 10^{-8}$ & $1.67 \times 10^{-8}$ & $5.45 \times 10^{-9}$ & $8.52 \times 10^{-9}$ & $1.90 \times 10^{-8}$ & $1.15 \times 10^{-8}$ & $2.80 \times 10^{-9}$ & $2.86 \times 10^{-9}$ & $1.00 \times 10^{-8}$ & $6.07 \times 10^{-9}$ \\
\hline & $7.75 \times 10^{-12}$ & $3.37 \times 10^{-12}$ & $1.31 \times 10^{-12}$ & $1.79 \times 10^{-12}$ & $4.38 \times 10^{-12}$ & $2.93 \times 10^{-12}$ & $7.75 \times 10^{-13}$ & $7.75 \times 10^{-13}$ & $3.00 \times 10^{-12}$ & $1.65 \times 10^{-12}$ \\
\hline & $4.48 \times 10^{-9}$ & $1.12 \times 10^{-8}$ & $3.90 \times 10^{-9}$ & $5.37 \times 10^{-9}$ & $1.43 \times 10^{-8}$ & $5.18 \times 10^{-9}$ & $5.48 \times 10^{-10}$ & $3.18 \times 10^{-10}$ & $3.28 \times 10^{-10}$ & $4.48 \times 10^{-10}$ \\
\hline $39 \mathrm{Ar}$ & $9.63 \times 10^{-10}$ & $2.55 \times 10^{-9}$ & $8.85 \times 10^{-10}$ & $1.22 \times 10^{-9}$ & $3.25 \times 10^{-9}$ & $1.19 \times 10^{-9}$ & $1.19 \times 10^{-10}$ & $6.69 \times 10^{-11}$ & $5.92 \times 10^{-11}$ & $1.02 \times 10^{-10}$ \\
\hline Err. ${ }^{39} \mathrm{Ar}$ & $8.93 \times 10^{-13}$ & $2.00 \times 10^{-12}$ & $8.29 \times 10^{-13}$ & $1.10 \times 10^{-12}$ & $2.51 \times 10^{-12}$ & $1.06 \times 10^{-12}$ & $5.13 \times 10^{-13}$ & $5.41 \times 10^{-13}$ & $4.74 \times 10^{-13}$ & $4.77 \times 10^{-13}$ \\
\hline$\%{ }^{3 y} \mathrm{Ar}$ & 9.26 & 24.52 & 8.51 & 11.71 & 31.26 & 11.41 & 1.15 & 0.64 & 0.57 & 0.98 \\
\hline 38Ar & $1.44 \times 10^{-10}$ & $3.17 \times 10^{-10}$ & $1.07 \times 10^{-10}$ & $1.41 \times 10^{-10}$ & $3.91 \times 10^{-10}$ & $1.53 \times 10^{-10}$ & $1.64 \times 10^{-11}$ & $9.81 \times 10^{-12}$ & $1.34 \times 10^{-11}$ & $1.55 \times 10^{-11}$ \\
\hline \multirow{2}{*}{$\begin{array}{l}\text { Err. }{ }^{38} \mathrm{Ar} \\
{ }^{38} \mathrm{ArCl}\end{array}$} & $2.25 \times 10-13$ & $5.06 \times 10^{-13}$ & $1.64 \times 10^{-13}$ & $2.21 \times 10^{-13}$ & $6.14 \times 10^{-13}$ & $2.39 \times 10^{-13}$ & $2.93 \times 10^{-14}$ & $2.03 \times 10^{-14}$ & $2.43 \times 10^{-14}$ & $2.84 \times 10^{-14}$ \\
\hline & $1.16 \times 10^{-10}$ & $2.85 \times 10^{-10}$ & $9.57 \times 10^{-11}$ & $1.25 \times 10^{-10}$ & $3.51 \times 10^{-10}$ & $1.36 \times 10^{-10}$ & $1.36 \times 10^{-11}$ & $7.46 \times 10^{-12}$ & $6.60 \times 10^{-12}$ & $1.08 \times 10^{-11}$ \\
\hline $37 \mathrm{Ar}$ & $1.18 \times 10^{-11}$ & $6.00 \times 10^{-12}$ & $9.43 \times 10^{-13}$ & $1.90 \times 10^{-12}$ & $1.66 \times 10^{-12}$ & $1.16 \times 10^{-12}$ & $4.95 \times 10^{-13}$ & $6.30 \times 10^{-13}$ & $1.32 \times 10^{-12}$ & $1.64 \times 10^{-12}$ \\
\hline Err. ${ }^{37} \mathrm{Ar}$ & $7.96 \times 10^{-14}$ & $7.54 \times 10^{-14}$ & $7.25 \times 10^{-14}$ & $7.29 \times 10^{-14}$ & $7.30 \times 10^{-14}$ & $7.28 \times 10^{-14}$ & $7.24 \times 10^{-14}$ & $7.25 \times 10^{-14}$ & $7.27 \times 10^{-14}$ & $7.27 \times 10^{-14}$ \\
\hline 36 $\mathrm{Ar}$ & $9.04 \times 10^{-11}$ & $1.84 \times 10^{-11}$ & $5.22 \times 10^{-12}$ & $1.06 \times 10^{-11}$ & $1.59 \times 10^{-11}$ & $2.13 \times 10^{-11}$ & $7.55 \times 10^{-12}$ & $8.51 \times 10^{-12}$ & $3.24 \times 10^{-11}$ & $1.88 \times 10^{-11}$ \\
\hline Err. ${ }^{36} \mathrm{Ar}$ & $2.64 \times 10^{-13}$ & $5.76 \times 10^{-14}$ & $2.00 \times 10^{-14}$ & $3.58 \times 10^{-14}$ & $5.11 \times 10^{-14}$ & $6.69 \times 10^{-14}$ & $2.54 \times 10^{-14}$ & $2.84 \times 10^{-14}$ & $9.61 \times 10^{-14}$ & $5.75 \times 10^{-14}$ \\
\hline Age & 8.45 & 8.00 & 7.99 & 8.00 & 7.96 & 7.93 & 8.32 & 8.63 & 10.07 & 7.99 \\
\hline Err. age $(1 \sigma)$ & 0.15 & 0.01 & 0.01 & 0.02 & 0.01 & 0.03 & 0.12 & 0.24 & 0.89 & 0.31 \\
\hline $\mathrm{Ca} / \mathrm{K}$ & 0.02379 & 0.00457 & 0.00207 & 0.00302 & 0.00099 & 0.00190 & 0.00804 & 0.01826 & 0.04316 & 0.03119 \\
\hline Err. $\mathrm{Ca} / \mathrm{K}$ & 0.00016 & 0.00006 & 0.00016 & 0.00012 & 0.00004 & 0.00012 & 0.00118 & 0.00211 & 0.00241 & 0.00139 \\
\hline $\mathrm{Cl} / \mathrm{K}$ & 0.02152 & 0.01989 & 0.01926 & 0.01832 & 0.01924 & 0.02039 & 0.02030 & 0.01985 & 0.01985 & 0.01895 \\
\hline Err. Cl/K & 0.00002 & 0.00002 & 0.00002 & 0.00002 & 0.00002 & 0.00002 & 0.00013 & 0.00025 & 0.00025 & 0.00014 \\
\hline${ }^{39} \mathrm{Ar} /{ }^{40} \mathrm{Ar}$ & 0.03060 & 0.15231 & 0.16225 & 0.14283 & 0.17096 & 0.10286 & 0.04261 & 0.02339 & 0.00591 & 0.01678 \\
\hline Err. ${ }^{39} \mathrm{Ar} /{ }^{40} \mathrm{Ar}$ & $2.94 \times 10^{-5}$ & $2.47 \times 10^{-4}$ & $3.14 \times 10^{-4}$ & $2.65 \times 10^{-4}$ & $2.76 \times 10^{-4}$ & $1.91 \times 10^{-4}$ & $1.83 \times 10^{-4}$ & $1.89 \times 10^{-4}$ & $4.74 \times 10^{-5}$ & $7.88 \times 10^{-5}$ \\
\hline${ }^{30} \mathrm{Ar} /{ }^{40} \mathrm{Ar}$ & 0.00287 & 0.00110 & 0.00096 & 0.00124 & 0.00084 & 0.00184 & 0.00270 & 0.00298 & 0.00324 & 0.00310 \\
\hline Err. ${ }^{36} \mathrm{Ar} /{ }^{40} \mathrm{Ar}$ & $8.42 \times 10^{-6}$ & $6.90 \times 10^{-6}$ & $7.36 \times 10^{-6}$ & $8.43 \times 10^{-6}$ & $5.39 \times 10^{-6}$ & $1.16 \times 10^{-5}$ & $9.08 \times 10^{-6}$ & $9.97 \times 10^{-6}$ & $9.65 \times 10^{-6}$ & $9.52 \times 10^{-6}$ \\
\hline
\end{tabular}

Analytical data show step number, step temperature, ${ }^{40}$ Ar total, ${ }^{40}$ Ar uncertainty, radiogenic ${ }^{40} \mathrm{Ar},{ }^{39} \mathrm{Ar},{ }^{39} \mathrm{Ar}$ uncertainty, ${ }^{39}$ Ar percentage, ${ }^{38} \mathrm{Ar},{ }^{38} \mathrm{Ar}$ uncertainty, ${ }^{38}$ ArCl, ${ }^{37} \mathrm{Ar},{ }^{37} \mathrm{Ar}$ uncertainty,

${ }^{36} \mathrm{Ar},{ }^{36} \mathrm{Ar}$ uncertainty, step age, age uncertainty as $1 \sigma, \mathrm{Ca} / \mathrm{K}$ ratio and its uncertainty, $\mathrm{Cl} / \mathrm{K}$ ratio and its uncertainty, ${ }^{39} \mathrm{Ar} /{ }^{40} \mathrm{Ar},{ }^{39} \mathrm{Ar} /{ }^{40} \mathrm{Ar}$ uncertainty, ${ }^{36} \mathrm{Ar} /{ }^{40} \mathrm{Ar},{ }^{36} \mathrm{Ar} /{ }^{40} \mathrm{Ar}$ uncertainty 
Table $9{ }^{39} \mathrm{Ar}^{-}{ }^{40} \mathrm{Ar}$ dating results of MH-T4 muscovite phenocrysts

MH-T4 muscovite

Weight $=0.01516 \mathrm{~g} ; \mathrm{J}=0.001006 ;[\mathrm{K}]=9.14 \% ;[\mathrm{Ca}]=630$ ppm; $[\mathrm{Cl}]=120$ ppm; Err. = Error

\begin{tabular}{|c|c|c|c|c|c|c|c|c|c|c|}
\hline Step & 1 & 2 & 3 & 4 & 5 & 6 & 7 & 8 & 9 & 10 \\
\hline $\mathrm{T}\left({ }^{\circ} \mathrm{C}\right)$ & 745 & 765 & 783 & 804 & 827 & 857 & 892 & 948 & 1134 & 1310 \\
\hline${ }^{40}$ Ar total & $3.15 \times 10^{-8}$ & $6.32 \times 10^{-9}$ & $7.77 \times 10^{-9}$ & $7.11 \times 10^{-9}$ & $5.56 \times 10^{-9}$ & $7.46 \times 10^{-9}$ & $8.21 \times 10^{-9}$ & $8.56 \times 10^{-9}$ & $1.04 \times 10^{-8}$ & $9.50 \times 10^{-9}$ \\
\hline Err. ${ }^{40} \mathrm{Ar}$ & $9.44 \times 10^{-12}$ & $1.28 \times 10^{-12}$ & $1.62 \times 10^{-12}$ & $1.92 \times 10^{-12}$ & $1.65 \times 10^{-12}$ & $3.37 \times 10^{-12}$ & $2.06 \times 10^{-12}$ & $1.92 \times 10^{-12}$ & $2.63 \times 10^{-12}$ & $2.16 \times 10^{-12}$ \\
\hline${ }^{40} \mathrm{Ar} *$ & $1.37 \times 10^{-9}$ & $1.57 \times 10^{-9}$ & $4.40 \times 10^{-9}$ & $5.26 \times 10^{-9}$ & $4.16 \times 10^{-9}$ & $5.56 \times 10^{-9}$ & $6.02 \times 10^{-9}$ & $5.98 \times 10^{-9}$ & $6.38 \times 10^{-9}$ & $2.10 \times 10^{-9}$ \\
\hline 39 $\mathrm{Ar}$ & $3.34 \times 10^{-10}$ & $3.54 \times 10^{-10}$ & $1.00 \times 10^{-9}$ & $1.20 \times 10^{-9}$ & $9.49 \times 10^{-10}$ & $1.27 \times 10^{-9}$ & $1.37 \times 10^{-9}$ & $1.36 \times 10^{-9}$ & $1.44 \times 10^{-9}$ & $4.65 \times 10^{-10}$ \\
\hline Err. ${ }^{39} \mathrm{Ar}$ & $5.21 \times 10^{-13}$ & $5.27 \times 10^{-13}$ & $9.24 \times 10^{-13}$ & $1.02 \times 10^{-12}$ & $8.52 \times 10^{-13}$ & $1.19 \times 10^{-12}$ & $1.10 \times 10^{-12}$ & $1.09 \times 10^{-12}$ & $1.17 \times 10^{-12}$ & $5.36 \times 10^{-13}$ \\
\hline$\%{ }^{3 y} \mathrm{Ar}$ & 3.43 & 3.64 & 10.29 & 12.33 & 9.73 & 13.04 & 14.09 & 13.92 & 14.75 & 4.77 \\
\hline $38 \mathrm{Ar}$ & $8.95 \times 10^{-11}$ & $8.03 \times 10^{-12}$ & $1.42 \times 10^{-11}$ & $1.53 \times 10^{-11}$ & $1.20 \times 10^{-11}$ & $1.60 \times 10^{-11}$ & $1.75 \times 10^{-11}$ & $1.76 \times 10^{-11}$ & $1.97 \times 10^{-11}$ & $1.03 \times 10^{-11}$ \\
\hline Err. ${ }^{38} \mathrm{Ar}$ & $1.29 \times 10-13$ & $1.76 \times 10^{-14}$ & $2.59 \times 10^{-14}$ & $3.04 \times 10^{-14}$ & $2.39 \times 10^{-14}$ & $2.75 \times 10^{-14}$ & $3.17 \times 10^{-14}$ & $3.26 \times 10^{-14}$ & $3.73 \times 10^{-14}$ & $1.95 \times 10^{-14}$ \\
\hline${ }^{38} \mathrm{Ar} C l$ & $6.68 \times 10^{-11}$ & $1.09 \times 10^{-12}$ & $9.02 \times 10^{-13}$ & $7.49 \times 10^{-13}$ & $5.84 \times 10^{-13}$ & $6.86 \times 10^{-13}$ & $8.82 \times 10^{-13}$ & $9.03 \times 10^{-13}$ & $1.20 \times 10^{-12}$ & $4.57 \times 10^{-13}$ \\
\hline 37 $\mathrm{Ar}$ & $1.55 \times 10^{-11}$ & $3.51 \times 10^{-12}$ & $2.48 \times 10^{-12}$ & $1.92 \times 10^{-12}$ & $1.44 \times 10^{-12}$ & $1.68 \times 10^{-12}$ & $1.41 \times 10^{-12}$ & $1.48 \times 10^{-12}$ & $2.67 \times 10^{-12}$ & $2.82 \times 10^{-12}$ \\
\hline Err. ${ }^{37} \mathrm{Ar}$ & $8.05 \times 10^{-14}$ & $7.19 \times 10^{-14}$ & $7.17 \times 10^{-14}$ & $7.16 \times 10^{-14}$ & $7.10 \times 10^{-14}$ & $7.11 \times 10^{-14}$ & $7.11 \times 10^{-14}$ & $7.12 \times 10^{-14}$ & $7.14 \times 10^{-14}$ & $7.14 \times 10^{-14}$ \\
\hline 36 $\mathrm{Ar}$ & $1.01 \times 10^{-10}$ & $1.59 \times 10^{-11}$ & $1.13 \times 10^{-11}$ & $6.18 \times 10^{-12}$ & $4.68 \times 10^{-12}$ & $6.36 \times 10^{-12}$ & $7.34 \times 10^{-12}$ & $8.65 \times 10^{-12}$ & $1.34 \times 10^{-11}$ & $2.48 \times 10^{-11}$ \\
\hline Err. ${ }^{36} \mathrm{Ar}$ & $2.72 \times 10-13$ & $4.61 \times 10^{-14}$ & $3.45 \times 10^{-14}$ & $2.08 \times 10^{-14}$ & $1.76 \times 10^{-14}$ & $2.20 \times 10^{-14}$ & $2.56 \times 10^{-14}$ & $2.75 \times 10^{-14}$ & $4.01 \times 10^{-14}$ & $7.04 \times 10^{-14}$ \\
\hline Age & 7.41 & 8.02 & 7.93 & 7.92 & 7.94 & 7.93 & 7.94 & 7.97 & 8.03 & 8.17 \\
\hline Err. age $(1 \sigma)$ & 0.44 & 0.07 & 0.02 & 0.01 & 0.01 & 0.01 & 0.01 & 0.01 & 0.02 & 0.08 \\
\hline $\mathrm{Ca} / \mathrm{K}$ & 0.08990 & 0.01922 & 0.00480 & 0.00310 & 0.00293 & 0.00256 & 0.00198 & 0.00212 & 0.00360 & 0.01176 \\
\hline Err. $\mathrm{Ca} / \mathrm{K}$ & 0.00049 & 0.00039 & 0.00014 & 0.00012 & 0.00015 & 0.00011 & 0.00010 & 0.00010 & 0.00010 & 0.00030 \\
\hline $\mathrm{Cl} / \mathrm{K}$ & 0.03557 & 0.00055 & 0.00016 & 0.00011 & 0.00011 & 0.00010 & 0.00011 & 0.00012 & 0.00015 & 0.00018 \\
\hline Err. $\mathrm{Cl} / \mathrm{K}$ & 0.00007 & 0.00004 & 0.00001 & 0.00001 & 0.00001 & 0.00001 & 0.00001 & 0.00001 & 0.00001 & 0.00003 \\
\hline${ }^{39} \mathrm{Ar} /{ }^{40} \mathrm{Ar}$ & 0.01061 & 0.05608 & 0.12912 & 0.16919 & 0.17063 & 0.17030 & 0.16728 & 0.15864 & 0.13876 & 0.04897 \\
\hline Err. ${ }^{39} \mathrm{Ar} /{ }^{40} \mathrm{Ar}$ & $1.69 \times 10^{-5}$ & $8.42 \times 10^{-5}$ & $1.22 \times 10^{-4}$ & $1.51 \times 10^{-4}$ & $1.61 \times 10^{-4}$ & $1.77 \times 10^{-4}$ & $1.40 \times 10^{-4}$ & $1.32 \times 10^{-4}$ & $1.18 \times 10^{-4}$ & $5.75 \times 10^{-5}$ \\
\hline${ }^{30} \mathrm{Ar} /{ }^{40} \mathrm{Ar}$ & 0.00320 & 0.00252 & 0.00145 & 0.00087 & 0.00084 & 0.00085 & 0.00089 & 0.00101 & 0.00129 & 0.00261 \\
\hline Err. ${ }^{36} \mathrm{Ar} /{ }^{40} \mathrm{Ar}$ & $8.70 \times 10^{-6}$ & $7.31 \times 10^{-6}$ & $4.44 \times 10^{-6}$ & $2.93 \times 10^{-6}$ & $3.17 \times 10^{-6}$ & $2.97 \times 10^{-6}$ & $3.12 \times 10^{-6}$ & $3.22 \times 10^{-6}$ & $3.88 \times 10^{-6}$ & $7.44 \times 10^{-6}$ \\
\hline
\end{tabular}

Analytical data show step number, step temperature, ${ }^{40} \mathrm{Ar}$ total, ${ }^{40} \mathrm{Ar}$ uncertainty, radiogenic ${ }^{40} \mathrm{Ar},{ }^{39} \mathrm{Ar},{ }^{39} \mathrm{Ar}$ uncertainty, ${ }^{39} \mathrm{Ar}$ percentage, ${ }^{38} \mathrm{Ar},{ }^{38} \mathrm{Ar}$ uncertainty, ${ }^{38} \mathrm{ArCl},{ }^{37} \mathrm{Ar},{ }^{37} \mathrm{Ar}$ uncertainty,

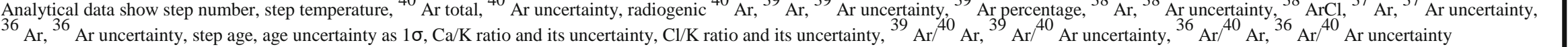

它


$\mathrm{Cl} / \mathrm{K}<0.00016$ (Fig. 7b, c). The isochron has an atmospheric intercept; therefore, it is valid to consider the weighted average age of steps 3-9, i.e., $7.95 \pm 0.03 \mathrm{Ma}$. All the ${ }^{39} \mathrm{Ar}-{ }^{40} \mathrm{Ar}$ data for both the biotite and muscovite separates are shown in Tables 8 and 9, respectively.

\section{Discussion}

The East Pisco Basin tephra of the P2 allomember are prevalently rhyolitic for what concerns the composition of glass shards (Bosio et al. 2019). The biotite composition of these tephra straddles the phlogopite and biotite fields, with a trend pointing to the siderophyllite end-member (see Fig. 5a). When plotted in the discrimination diagrams for granitic rocks based on biotite composition (Fig. 5c, d), they mostly fall in the field corresponding to mica in calc-alkaline rocks, except for a few falling in the field of mica in peraluminous rocks. Among these last tephra, one contains juvenile muscovite, biotite, and $\mathrm{Al}_{2} \mathrm{SiO}_{5}$ silicates, revealing a strongly peraluminous composition of the erupted magma. The presence of this peculiar mineral association, unusual in subduction-related arc system, leads to discuss the possible volcanic source for the ash.

Two-mica volcanic rocks are rare in the geological record, and examples in upper Miocene can be found in the Eastern Cordillera, which host one of the largest peraluminous provinces in the world (Sandeman et al. 1997). In the Eastern Cordillera, the presence of volcanic muscovite has only been reported in the Macusani and Morococala SP volcanic products (Noble et al. 1984; Morgan et al. 1998). The MiocenePliocene Macusani ignimbrite field is the largest SP volcanic unit in southern Peru (Noble et al. 1984; Pichavant et al. 1988a, b; Cheilletz et al. 1992). This field comprises crystalrich ash-flow tuffs and rare obsidian glasses, with an unusual mineralogy similar to two-mica peraluminous leucogranites (Pichavant et al. 1987, 1988a). The Macusani Formation consists of a 500-m-thick package of ash flows of variable thickness, comprising six major cooling units, which emplaced into two volcanic cycles, giving origin to a Lower and an Upper Member of the formation (Cheilletz et al. 1992). In the Eastern Cordillera of Bolivia, the late Miocene Morococala volcanic field is composed of andalusite-, biotite-, and muscovite-bearing rhyolite tuffs at the base, cordierite- and biotite-bearing rhyolite tuffs, and biotite-bearing quartz latite tuffs and lavas at the top (Morgan et al. 1998).

A correlation with these Eastern Cordillera volcanics can be discussed on the basis of the mineral assemblage and chem-istry, glass composition, and age determinations. The chemi-cal composition of biotite, which has been demonstrated to be a useful tool for tephra correlations (Shane et al. 2003; Lebti et al. 2006; Bosio et al. 2019), allows discriminating among the peraluminous Central Andes volcanics (Caffe et al. 2012).
The biotite composition of the MH-T4 and CLQ-T23/LB-T19 tephra layers corresponds well with analyses of mica of the Macusani and Morococala SP volcanic units from previous work (i.e., Noble et al. 1984; Pichavant et al. 1988a, b; Morgan et al. 1998), except for the low fluorine content (see Tables 1 and 2; Fig. 5). In the case of MH-T4 ash layer, the correlation is supported by the high $\mathrm{F}$ and the major and minor element composition of muscovite and by the composition of feldspar and Al-silicate minerals (Tables 3, 4, and 5).

To compare the glass composition of the Pisco tephra with the Macusani and Morococala volcanics, the available data for matrix glasses and obsidian glasses (macusanite) from previ-ous work (Pichavant et al. 1987; London et al. 1988; Morgan et al. 1998) are reported in Table 6 and Fig. 6. A correlation with Macusani and Morococala volcanic regions is supported by the major element composition of matrix glass and by the high $\mathrm{F}$ content measured on the MH-T4 glass shards. The trace element composition of the ash glasses, with particularly high $\mathrm{B}$, shows a striking correspondence with the Macusani matrix glasses and a rather similar pattern with the Morococala glass inclusions. The major discrepancy is shown by $\mathrm{Li}$, which is very low in the East Pisco Basin tephra (Table 7, Fig. 6e). Lithium, however, is a highly mobile ele-ment, and volcanic glass may be depleted in Li during post-depositional processes, as recently reported in several studies (e.g., Misra and Froelich 2012; Hofstra et al. 2013; Ellis et al. 2018).

The age of the MH-T4 tephra eruption was determined on biotite and muscovite due to the fact that the consistent mineral assemblage and composition indicate that the ash layer represents deposition from a single eruption, with both muscovite and biotite as juvenile phenocrysts. The quantitative compositional data indicate that submarine alteration was not pervasive, allowing us to determine the age of the erup-tion. The ${ }^{39} \mathrm{Ar}-{ }^{40} \mathrm{Ar}$ ages determined separately on the two micas are identical, $7.95 \pm 0.03 \mathrm{Ma}$ and $7.98 \pm 0.03 \mathrm{Ma}$, and allow us to propose a weighted average age of $7.96 \pm 0.02 \mathrm{Ma}$ for this eruption. This age is more reliable and precise than the previous age of $8.05 \pm 0.14 \mathrm{Ma}$ obtained from biotite by Bosio et al. (2020b); biotite shows a lower Ca concentration than the previous sample, which indicates a reduced amount of Ca-rich impurities/alteration phases (Fig 7b).

The age of $7.96 \pm 0.02 \mathrm{Ma}$ falls within the literature age range of the Peruvian Macusani volcanics, between ca. 10 and $4 \mathrm{Ma}$ (Pichavant et al. 1988a; Cheilletz et al. 1992; Poupeau et al. 1993) and with the late Miocene ages of the Bolivian Morococala volcanic field (Koeppen et al. 1987; Morgan et al. 1998). According to Cheilletz et al. (1992), the Macusani erup-tion ages (i.e., $10.0 \pm 0.5 \mathrm{Ma}, 7.8-8.0 \pm 0.1 \mathrm{Ma}, 7.5 \pm 0.1 \mathrm{Ma}, 7.3 \pm 0.1$ $\mathrm{Ma}, 6.8-7.0 \pm 0.1 \mathrm{Ma}, 6.7 \pm 0.1 \mathrm{Ma})$ cluster in corre-spondence to two important Andean tectonic phases, with the second cluster being by far the most voluminous. These ages are in agreement with the K-Ar ages reported by Pichavant 
et al. (1988a), i.e., 10.5 $\pm 0.5 \mathrm{Ma}, 7.6 \pm 0.3 \mathrm{Ma}, 7.1 \pm 0.7 \mathrm{Ma}$, and $6.8 \pm 0.2 \mathrm{Ma}$, and with those proposed by Poupeau et al. (1993), i.e., $7 \pm 1 \mathrm{Ma}$ and late episodes at 5.7-5.3 and 4.8-4.3 Ma. The late Miocene ages of 7.6-8 Ma for Macusani agree with the $7.96 \mathrm{Ma}$ age of the two-mica tephra. MH-T4 age also overlaps with the Morococala late Miocene ages. According to ${ }^{39} \mathrm{Ar}-{ }^{40} \mathrm{Ar}$ dating, the andalusite-bearing rhyolites give an age of ca. 8.4 Ma, the cordierite-bearing rhyolites $6.8 \mathrm{Ma}$, and the quartz latites 6.4 Ma (Koeppen et al. 1987). Since the twomica volcanic prod-ucts are only present in the andalusitebearing rhyolites, only the age of this unit should be taken into account for comparison with the Pisco tephra. This age is consistent with the $7.96 \pm 0.02 \mathrm{Ma}$ age of MH-T4.

The CLQ-T23/LB-T19 tephra can be traced and recognized by tephra fingerprinting and correlation for over 6 $\mathrm{km}$ at both the localities of Cerro los Quesos (CLQ-T23) and Cerro la Bruja (LB-T19) (Bosio et al. 2019). The age, indi-rectly bracketed by ${ }^{39} \mathrm{Ar}-{ }^{40} \mathrm{Ar}$ dating of ash layers in the same stratigraphic sections (Di Celma et al. 2017; Gariboldi et al. 2017), is between $7.45 \pm 0.01 \mathrm{Ma}$ and 6.93 $\pm 0.09 \mathrm{Ma}$. Direct age determination could not be achieved here because of the small size of biotite phenocrysts. This late Miocene age over-laps with the late Miocene eruptions of both the Macusani and Morococala volcanic fields (Cheilletz et al. 1992; Koeppen et al. 1987).

The characteristics of the MH-T4 and CLQ-T23/LB-T19 tephra, such as the grain-size classification as extremely fine ash following White and Houghton (2006), correspond to deposition of distal ash from fallout from Plinian plume-cloud systems or co-ignimbrite plumes rising above pyroclastic density currents. The good correspondence of the mineral assemblage, composition of mineral and glass phases, and the smaller distance from vents suggest to propose a correlation of MH-T4 tephra with the ignimbrites of Macusani. We suggest that a major eruption of the Macusani field occurred at 7.96 $\mathrm{Ma}$ and that the MH-T4 layer could correspond to one of the largest events of the Macusani region, with ash deposited at over $500 \mathrm{~km}$ of distance.

These results indicate that the coastal basins of Peru are highly promising as archives for distal ash from Central Andes eruptions and supereruptions, not only from the Western Cordillera and Altiplano but also from the Eastern Cordillera. Future studies may lead to a better reconstruction of the volcanic history of the Central Andes of Southern Peru.

\section{Conclusion}

Few strongly peraluminous volcanic eruptions are reported in the Central Andes and, among these, two-mica volcanics are particularly rare. The mineral assemblage and the mineral and glass composition of the two-mica Pisco ash correspond to the Macusani volcanic complex, active between 10 and 4 Ma in the Eastern Cordillera of Peru, and to the late Miocene Morococala, in northern Bolivia. Concordant biotite and muscovite age determinations indicate an age of $7.96 \mathrm{Ma} \pm 0.02$ Ma. This age, together with the petrographic correspon-dence and the smaller distance between source and sample, leads us to suggest a correlation with the Macusani volcanic field. These results provide new data on the volcanic activity of the Central Andes, suggesting that a major explosive erup-tion at $7.96 \mathrm{Ma}$, probably from the Macusani field. This re-sulted in the dispersal of co-ignimbrite ash to over $500 \mathrm{~km}$ to the west and into the forearc marine basins. This finding pos-sibly represents the farthest occurrence of Eastern Cordillera volcanics in the geological record and highlights the importance of the exhumed forearc basins as an archive of distal ash from Central Andes volcanoes.

Acknowledgments The authors are grateful to R. Varas-Malca and W. Aguirre for their assistance in the field and at the Natural History Museum in Lima. Special thanks to E. Malinverno for the support in the field and in laboratory analyses, to V. Barberini for the help in ${ }^{39} \mathrm{Ar}-{ }^{40} \mathrm{Ar}$ dating, and N. Fusi for grain-size analyses. The authors wish to thank A. Risplendente for the help with the microprobe analyses. Finally, the authors would like to thank two anonymous reviewers, V.S. Kamenetsky and the Executive Editor for their revisions and thorough support.

Funding This study was supported by grants from the Italian Ministero dell'Istruzione dell'Università e della Ricerca (PRIN Project 2012YJSBMK) and by the University of Pisa PRA_2015_0028 and PRA_2017_0032 grants.

\section{References}

Abdel-Rahman AM (1994) Nature of biotites from alkaline, calcalkaline, and peraluminous magmas. J Petrol 35:525-554

Baker MCW, Francis PW (1978) Upper Cenozoic volcanism in the Central Andes-ages and volumes. Earth Planet Sci Lett 41(2): 175-187

Bianucci G, Di Celma C, Collareta A, Landini W, Post K, Tinelli C, de Muizon C, Bosio G, Gariboldi K, Gioncada A, Malinverno E, Cantalamessa G, Altamirano-Sierra A, Salas-Gismondi R, Urbina M, Lambert O (2016a) Fossil marine vertebrates of Cerro Los Quesos: distribution of cetaceans, seals, crocodiles, seabirds, sharks, and bony fish in a late Miocene locality of the Pisco Basin, Peru. J Maps 12:1037-1046

Bianucci G, Di Celma C, Landini W, Post K, Tinelli C, de Muizon C, Gariboldi K, Malinverno E, Cantalamessa G, Gioncada A, Collareta A, Salas-Gismondi R, Varas-Malca RM, Urbina M, Lambert O (2016b) Distribution of fossil marine vertebrates in Cerro Colorado, the type locality of the giant raptorial sperm whale Livyatan melvillei (Miocene, Pisco Formation, Peru). J Maps 12: 543-557

Bianucci G, Bosio G, Malinverno E, de Muizon C, Villa IM, Urbina M, Lambert O (2018) A new large squalodelphinid (Cetacea, Odontoceti) from Peru sheds light on the Early Miocene platanistoid disparity and ecology. R Soc Open Sci 5:172302

Blott SJ, Pye K (2001) Gradistat: a grain size distribution and statistics package for the analysis of unconsolidated sediments. Earth Surf Process Landf 26:1237-1248 
Bosio G, Gioncada A, Malinverno E, Di Celma C, Villa IM, Cataldi G, Gariboldi K, Collareta A, Urbina M, Bianucci G (2019) Chemical and petrographic fingerprinting of volcanic ashes as a tool for high-resolution stratigraphy of the upper Miocene Pisco Formation (Peru). J Geol Soc Lond 176:13-28

Bosio G, Malinverno E, Collareta A, Di Celma C, Gioncada A, Parente M, Berra F, Marx FG, Vertino A, Urbina M, Bianucci G (2020a) Strontium isotope stratigraphy and the thermophilic fossil fauna from the middle Miocene of the East Pisco Basin (Peru). J S Am Earth Sci 97:102399

Bosio G, Malinverno E, Villa IM, Di Celma C, Gariboldi K, Gioncada A, Barberini V, Urbina M, Bianucci G (2020b) Tephrochronology and chronostratigraphy of the Miocene Chilcatay and Pisco formations (East Pisco Basin, Peru). Newsl Stratigr 53(2):213-247. https://doi. org/10.1127/nos/2019/0525

Breitkreuz C, de Silva SL, Wilke HG, Pfander JA, Renno AD (2014) Neogene to quaternary ash deposits in the Coastal Cordillera in northern Chile: distal ashes from supereruptions in the Central Andes. J Volcanol Geotherm Res 269:68-82

Caffe PJ, Trumbull RB, Siebel W (2012) Petrology of the Coyaguayma ignimbrite, northern Puna of Argentina: origin and evolution of a peraluminous high-SiO2 rhyolite magma. Lithos 134-135:179-200 Cheilletz A, Clark AH, Farrar E, Arroyo Pauca G, Pichavant M, Sandeman HA (1992) Volcano-stratigraphy and 40Ar/39Ar geochronology of the Macusani ignimbrite field: monitor of the Miocene geodynamic evolution of the Andes of Southeast Peru. Tectonophysics 205:307-327

Clarke DB (1981) The mineralogy of peraluminous granites; a review. Can Mineral 19:3-17

de Silva SL, Francis PW (1991) Volcanoes of the Central Andes. Springer-Verlag, New York

de Silva SL, Zielinski GA (1998) Global influence of the AD 1600 erup-tion of Huaynaputina, Peru. Nature 393(6684):455-458

Di Celma C, Malinverno E, Bosio G, Collareta A, Gariboldi K, Gioncada A, Molli G, Basso D, Varas-Malca R, Pierantoni PP, Villa IM, Lambert O, Landini W, Sarti G, Cantalamessa G, Urbina M, Bianucci G (2017) Sequence stratigraphy and palaeontology of the upper Miocene Pisco Formation along the western side of the lower Ica valley (Ica desert, Peru). Riv Ital Paleontol S 123:255-274

Di Celma C, Malinverno E, Bosio G, Gariboldi K, Collareta A, Gioncada A, Landini W, Pierantoni PP, Bianucci G (2018) Intraformational unconformities as a record of Late Miocene eustatic falls of sea level in the Pisco Formation (southern Peru). J Maps 14(2):607-619

Dunbar RB, Marty RC, Baker PA (1990) Cenozoic marine sedimentation in the Sechura and Pisco basins, Peru. Palaeogeogr Palaeocl 77:235- 261

Ellis BS, Szymanowski D, Magna T, Neukampf J, Dohmen R, Bachmann O, Ulmer P, Guillong M (2018) Post-eruptive mobility of lithium in volcanic rocks. Nat Commun 9:1-9

Esperante R, Brand LR, Chadwick AV, Poma O (2015) Taphonomy and paleoenvironmental conditions of deposition of fossil whales in the diatomaceous sediments of the Miocene/Pliocene Pisco Formation, southern Peru - a new fossil-lagerstätte. Palaeogeogr Palaeocl 417: 337-370

Gariboldi K, Bosio G, Malinverno E, Gioncada A, Di Celma C, Villa IM, Urbina M, Bianucci G (2017) Biostratigraphy, geochronology and sedimentation rates of the upper Miocene Pisco Formation at two important marine vertebrate fossilbearing sites of southern Peru. Newsl Stratigr 50(4):417-444

Griffin WL (2008) GLITTER: data reduction software for laser ablation ICP-MS. In: Sylvester P (ed) Laser ablation ICP-MS in the earth sciences: current practices and outstanding issues. Mineralogical Association of Canada, Vancouver BC, pp 308-311

Griggs AJ, Davies SM, Abbott PM, Rasmussen TL, Palmer AP (2014) Optimising the use of marine tephrochronology in the North
Atlantic: a detailed investigation of the Faroe Marine Ash Zones II, III and IV. Quat Sci Rev 106:122-139

Hart D, Miller DJ (2006) Analysis and correlation of volcanic ash in marine sediments from the Peru margin, Ocean Drilling Program Leg 201: explosive volcanic cycles of the northCentral Andes. In: Jørgensen BB, D’Hondt SL, Miller DJ (eds) Proceedings of the ocean drilling program, scientific results, 201. ODP, College Station, pp 1-43

Hofstra AH, Todorov TI, Mercer CN, Adams DT, Marsh EE (2013) Silicate Melt Inclusion Evidence for Extreme Pre-eruptive Enrichment and Post-eruptive Depletion of Lithium in Silicic Volcanic Rocks of the Western United States: Implications for the Origin of Lithium-Rich Brines. Econ Geol 108:1691-1701

Kay SM, Coira BL, Caffe PJ, Chen C-H (2010) Regional chemical diversity, crustal and mantle sources and evolution of central Andean Puna plateau ignimbrites. J Volcanol Geotherm Res 198:81-111

Koeppen RP, Smith RL, Kunk MJ, Flores AM, Luedke RG, Sutter JF (1987) The Morococala volcanics: highly peraluminous rhyolite ash flow magmatism in the Cordillera Oriental, Bolivia. Geol Soc Am Abstr Programs 19:731

Lebti PP, Thouret J-C, Worner G, Fornari M (2006) Neogene and quater-nary ignimbrites in the area of Arequipa, southern Peru: Stratigraphical and petrological correlations. J Volcanol Geotherm Res 154:251-275

London D, Hervig RL, Morgan GBVI (1988) Melt-vapor solubilities and elemental partitioning in peraluminous granite-pegmatite systems: experimental results with Macusani glass at $200 \mathrm{MPa}$. Contrib Mineral Petrol 99:360-373

Mamani M, Wörner G, Sempere T (2010) Geochemical variations in igneous rocks of the central Andean orocline $\left(13^{\circ} \mathrm{S}\right.$ to $\left.18^{\circ} \mathrm{S}\right)$ : tracing crustal thickening and magma generation through time and space. Geol Soc Am Bull 122:162-182

Miller CF (1985) Are strongly peraluminous magmas derived from pelitic sedimentary sources? J Geol 93:673-689

Misra S, Froelich PN (2012) Lithium isotope history of Cenozoic seawa-ter: changes in silicate weathering and reverse weathering. Science 335(6070):818-823

Mlynarczyk MSJ, Williams-Jones AE (2005) The role of collisional tec-tonics in the metallogeny of the Central Andean tin belt. Earth Planet Sci Lett 240:656-667

Morgan GBVI, London D, Luedke RG (1998) Petrochemistry of lateMiocene peraluminous silicic volcanic rocks from the Morococala field, Bolivia. J Petrol 39:601-632

Noble DC, Vogel TA, Peterson PS, Landis GP, Grant NK, Jezek PA, Mc Kee EH (1984) Rare-element enriched, S-type ash-flow tuffs con-taining phenocrysts of muscovite, andalusite and sillimanite, south-eastern Peru. Geology 12:35-39

Peccerillo A, Taylor SR (1976) Geochemistry of Eocene calcalkaline volcanic rocks from the Kastamonu area, northern Turkey. Contrib Mineral Petrol 58:63-81

Pichavant M, Herrera JV, Boulmier S, Briqueu L, Joron J-L, Juteau M, Marin L, Michard A, Sheppard SMF, Treuil M, Vernet M (1987) The Macusani glasses, SE Peru: evidence of chemical fractionation in peraluminous magmas. Geochem Soc Spec Publ 1:359-373

Pichavant M, Kontak DJ, Valencia Herrera J, Clark AH (1988a) The Miocene-Pliocene Macusani volcanics, SE Peru: I. mineralogy and magmatic evolution of a two-mica aluminosilicate bearing ig-nimbrite suite. Contrib Mineral Petrol 100:300-324

Pichavant M, Kontak DJ, Briqueu L, Valencia Herrera J, Johnston RM (1988b) The Miocene-Pliocene Macusani Volcanics, SE Peru: II. Geochemistry and origin of a felsic peraluminous magma. Contrib Mineral Petrol 100:325-338

Pouclet A, Cambray H, Cadet J-P, Bourgois J, De Wever P (1990) Volcanic ash from leg 112 off Peru. In: Suess E, von Huene R et al (eds) Proceedings of the ODP, scientific results, 112:465-480 
Poupeau G, Labrin E, Sabil N, Bigazzi G, Arroyo G, Vatin-Pérignon N (1993) Fission-track dating of 15 macusanite glass pebbles from the Macusani volcanic field (SE Peru). Nucl Tracks Rad Meas 21(4): 499-506

Rosenbaum G, Giles D, Saxon M, Betts PG, Weinberg RF, Duboz C (2005) Subduction of the Nazca Ridge and the Inca Plateau: insights into the formation of ore deposits in Peru. Earth Planet Sci Lett 239: 18-32

Sandeman HA, Clark AH (2003) Glass-rich, cordierite-biotite rhyodacite, Valle Ninahuisa, Puno, southeastern Peru: petrological evidence for hybridization of "Lachlan S-type" and potassic mafic magmas. J Petrol 44:355-385

Sandeman HA, Clark AH (2004) Commingling and mixing of S-type peraluminous, ultrapotassic and basalticmagmas in the Cayconi volcanic field, Cordillera de Carabaya, SE Peru. Lithos 73:186-213

Sandeman HA, Clark AH, Farrar E (1997) Lithostratigraphy, petrology and 40Ar-39Ar geochronology of the Crucero Supergroup, Puno department, SE Peru. J S Am Earth Sci 10:223-245

Schneider A (1987) Eruptive processes, mineralization and isotopic evo-lution of the Los Frailes, Kari Kari region, Bolivia. Rev Geol Chile 30:27-33

Shand SJ (1943) The eruptive rocks, 2nd edn. Wiley, New York

Shane P, Smith V, Nairn I (2003) Biotite composition as a tool for the identification of quaternary tephra beds. Quat Res 59:262-270
Tada R, Murray RW et al (2015) Methods. In: Tada R, Murray RW et al (eds) Proceedings of the IODP, expedition reports, College Station, Texas, 346:1-58

Taylor SR, McLennan SM (1985) The continental crust: its composition and evolution. Blackwell Science Inc, United States

Thornburg TM, Kulm LD (1981) Sedimentary basins of the Peru continental margin: Structure, stratigraphy, and Cenozoic tectonics from $6^{\circ} \mathrm{S}$ to $16^{\circ} \mathrm{S}$ latitude. In: Kulm LD, Dymond J, Dasch EJ, Hussong DM (eds) Nazca plate: Crustal formation and Andean convergence 393-422. Boulder: Geol Soc Am Mem 154

Thouret JC, Davila J, Eissen JP (1999) Largest explosive eruption in historical times in the Andes at Huaynaputina volcano, AD 1600, southern Peru. Geology 27(5):435-438

Travis RB, Gonzales G, Pardo A (1976) Hydrocarbon potential of coastal basins of Peru. Am Assoc Petr Geol Memoir 25:331-338

Trumbull RB, Riller U, Oncken O, Scheuber E, Munier K, Hongn F (2006) The time-space distribution of Cenozoic volcanism in the South-Central Andes: a new data compilation and some tectonic implications. In: Oncken $\mathrm{O}$ et al (eds) The Andes. Frontiers in Earth Sciences. Springer, Berlin, Heidelberg

Villa IM, Ruggieri G, Puxeddu M, Bertini G (2006) Geochronology and isotope transport systematics in a subsurface granite from the Larderello-Travale geothermal system (Italy). J Volcanol Geotherm Res 152:20-50

White JDL, Houghton BF (2006) Primary volcaniclastic rocks. Geology 34:677-680 